\title{
Planar Graphical Models which are Easy
}

\author{
Vladimir Y. Chernyak ${ }^{a, b}$ and Michael Chertkov ${ }^{b, c}$ \\ ${ }^{a}$ Department of Chemistry, Wayne State University, 5101 Cass Ave,Detroit, MI 48202 \\ ${ }^{b}$ Center for Nonlinear Studies and Theoretical Division, LANL, Los Alamos, NM 87545 \\ ${ }^{c}$ New Mexico Consortium, Los Alamos, NM 87544, USA
}

(Dated: October 30, 2018)

\begin{abstract}
We describe a rich family of binary variables statistical mechanics models on a given planar graph which are equivalent to Gaussian Grassmann Graphical models (free fermions) defined on the same graph. Calculation of the partition function (weighted counting) for such a model is easy (of polynomial complexity) as reducible to evaluation of a Pfaffian of a matrix of size equal to twice the number of edges in the graph. In particular, this approach touches upon Holographic Algorithms of Valiant [1-3] and utilizes the Gauge Transformations discussed in our previous works 448 .
\end{abstract}

PACS numbers: 02.50.Tt, 64.60.Cn, 05.50.+q

\section{INTRODUCTION}

This paper rests on classical results derived and discussed in statistical physics and computer science. Onsager solution [9] of the two-dimensional Ising model on a square grid, its combinatorial interpretation by Kac, Ward [10] and Vdovichenko [11, and the relation between the Ising model and the dimer model, established independently by Temperley, Fisher [12, 13] and Kasteleyn [14, 15, have aimed mainly at analysis of phase transitions in parametrically homogeneous infinite systems. However, the algebraic and combinatorial techniques used in these papers were intrinsically microscopic, and thus suitable for analysis of a much broader class of problems, e.g. these that are parametrically inhomogeneous (glassy) and are formulated on finite planar graphs. In fact, the approach was extended [13, 16] to inhomogeneous versions of these models formulated on an arbitrary finite planar graph of size $N$. It was shown that, under these conditions, the partition (generating) function of an arbitrary Ising model (however without magnetic field) or of the dimer model are represented by Pfaffians (or determinants) of the properly defined $N \times N$ matrices. Note that computation of a Pfaffian/determinant requires utmost $N^{3}$ steps, while calculation of the partition function for a generic statistical physics model is a task of likely exponential complexity. More accurately, this is the task of the \#P complexity class according to computer science classification, started with the classical papers of Cook [17. and Karp [18. The \#P feature of the generic statistical physics model makes the easiness of the planar Ising and dimer models surprising and exceptional, especially in view of the results of Barahona [19, who showed that adding magnetic field (even homogeneous) to the planar Ising model immediately elevates the weighted counting problem (this is the partition function computation) to be of the \#P-complete class, i.e. of the complexity necessary and sufficient for solving any other problem that belongs to the \#P hierarchy ${ }^{1}$. Similarly, adding monomers turns the dimer-monomer model on a planar graph (or even on a planar bi-partite graph) into a problem that belongs to the \#P-complete class $[21]^{2}$.

One wonders if the easiness of the dimer and Ising models on planar graphs is a lucky exception or may be it is, in fact, just a little top of yet unexplored iceberg? A new approach of Valiant [1] 3, coined by the author "holographic algorithm", shed some additional light onto this question. In [1 3 . Valiant described a list of easy planar models reducible to dimer models on planar graphs via a set of "gadgets". The gadgets were of "classical" and "holographic" types. A classical gadget describes an elementary graphical transformation, applied to the variables defined on a graph element, e.g. edge or a region, that preserves a one-to-one correspondence between the configurations of the original and transformed models. An example of a classical gadget would be an approach used by Fisher 13 (pointto-triangle transformation) to map the Ising model onto a dimer model. A holographic gadget of [1] involves a linear transformation of the parametrization basis for the binary variables, so that the solution fragments of the original and mapped models would be in a mixed relation when certain sums, rather than individual elements of the original and derived formulations, are related to each other. The freedom in choosing an arbitrary nonsingular basis for the holographic transformation was discussed in [1-3], however, it was explored there in a somewhat limited fashion. One emphasis of [1-3] was on generating a polynomial time algorithm (via reduction to a determinant) for a number of

\footnotetext{
1 The \#P, pronounced sharp-P, and \#P-complete classes were introduced by Valiant in [20], who studied the complexity of calculating the matrix permanent.

2 Dimer and monomer-dimer models from statistical physics are called perfect matching and matching models, respectively, in computer science.
} 
problems for which only exponential time algorithms where known before, such as counting the edge orientations on a planar graph of maximal degree 3 with no nodes containing all the edges directed towards or away from it. This model belongs to the class of "ice" problems studied earlier in statistical physics 22 24. We will actually discuss this example in details later in Section VC. A class of easy factor-function models, stated in terms of binary edge variables on planar graphs of degree not larger than three, was also discussed in [6]. These models were stated in terms of a set of algebraic equations, one per vertex, constraining the factor-functions of the model.

In this paper we present a family of statistical models, stated in terms of binary variables defined on the edges of a given planar graph, with the partition functions reducible to Pfaffians of square matrices of the size equal to twice the number of graph edges. The general model of this class (easy on a given planar graph) can be defined in two consecutive steps: (a) construct an arbitrary Wick Binary Graphical Model (WBG) on the graph, and (b) further apply an arbitrary Gauge transformation of the type discussed in our early work devoted to the Loop Calculus (LC) approach [4 8. Of the two steps, both required to characterize this class of easy binary models on a given graph, the description of the easy WBG models on a given planar graph and consecutively showing that it is Pfaffian (or det)-easy, is the most novel contribution of the paper. Therefore, we find it useful to informally describe a general WBG model already here in the Introduction. (See Section II A for the formal description.)

A WBG model is defined in terms of painting the graph edges (each edge can be colored or not) with the "even coloring" requirement (for each vertex the number of colored edges adjusted to it should be even). The weight of an allowed configuration (an even coloring) is given by the product of the vertex weights/contributions. A vertex contribution is equal to unity when no edges adjusted to the vertex are colored. The vertex weights are introduced for all pair colorings, defined as painting of pairs of edges adjusted to the vertex. Thus, the total number of unconstrained parameters associated with a vertex is $N_{v}\left(N_{v}-1\right) / 2$, where $N_{v}$ is the vertex degree (valence), i.e., the number of edges adjusted to the vertex. The weights of all higher-degree colorings $(4,6, \cdots$ edges of the vertex are colored) are not independent, but rather explicitly expressed in terms of the pair weights according to a simple rule, illustrated below using an example of a vertex of degree four. Assume that the edges of the degree four vertex are numbered clockwise $1,2,3,4$ with the corresponding weights of the pair-wise colorings being $w_{12}, w_{13}, w_{14}, w_{23}, w_{24}, w_{34}$. Then, the weight of the four-edge coloring is $w_{12} w_{34}-w_{13} w_{24}+w_{14} w_{23}$. We refer to the described rule and the corresponding model as the Wick rule/model, since modulo signs, the expression for the higher-order weights is represented by the Wick's decomposition of a higher-order (even) correlation functions of a Gaussian random field in terms of the pair correlation functions (covariances). The sign rule for an individual contribution (that corresponds to a partitioning the even number of $2 k$ colored edges into $k$ pairs) to the weight is defined as follows: (i) represent the edges by points on a circle (according to their cycling ordering), (ii) connect the points that represent the paired edges with lines, (iii) count the total number of the line crossings inside the circle, (iv) choose plus/minus sign if the number is even/odd. Note, that the Ising model, the dimer model and some other planar easy models discussed in 1 1 3, can be represented, after a gauge transformation, in terms of a special case of the general WBG model. For example, an WBG representation for the Ising model is directly evident from the respective high-temperature expansion/series. (See 25] and discussion of Section VD,

In this manuscript we show that

- The WBG model with an arbitrary choice of the edge associated weights, $w$, is equivalent to corresponding Fermion Gaussian model, referred to as the Grassman Gaussian Graphical $\left(\mathrm{G}^{3}\right)$ model, and defined on the same graph. With a suitable choice of the so-called complete orientations for edge and triplets of neighboring vertices contributing definition of the $\mathrm{G}^{3}$ model (and thus called in the following suitable complete orientation of the graph, or when it is not confusing simply -suitable orientation), the two models are equivalent in the sense that their partition functions are equal to each other and also equal to an explicitly defined Pfaffian of a skewsymmetric matrix of the size twice the number of edges in the graph. Obviously, computational complexity of the object is polynomial in the graph size. This main result of the manuscript is formally stated in the Theorem II.7.

- Application of an arbitrary and graph-local gauge transformation (described in terms of a pair of $2 \times 2$ matrices per edge orthogonal to each other) to the WBG model generates another det- easy model.

This paper builds upon, and in a certain sense extends, the classical results of Kasteleyn [15, 16] and Temperley, Fisher [12, 13] developed for a planar dimer model. One key ingredient of the Kastelyan approach was the construction of edge-orientations. It was shown that for a special choice of the edge-orientations, so-called Kasteleyn edge-orientations, the partition function of the dimer model becomes a Pfaffian of a skew symmetric matrix built from element-by-element product of the edge weights and the \pm 1 Kasteleyn edge-orientation factors. As shown in [15, 16], construction of the Kasteleyn orientation of a planar graph can be done efficiently in the number of steps linear in the size of the system. (See also Section 8.3 of [26.). Our scheme also requires defining graph-local orientations, however of an extended character, where not only edge-orientations, but also triplet-orientations are needed. The combination of 
the edge-orientations and triplet-orientations defined on a given graph will be called complete orientations. A special choice of the complete orientation, the suitable complete orientation, in a sense generalizes the notion of the Kasteleyn edge-orientation. See Section II for accurate definitions.

Another important graph-related structure utilized in this paper is the so-called even generalized loops, decomposed into combinations of embedded orbits on the graph. For any finite graph these graph objects are finite, which allows to represent relevant partition functions in terms of a sum over a finite number of contributions associated with embedded orbits. At this point it is also appropriate to refer to a related, yet different, approach that operates with (random) walks and associated immersed orbits, that has been formulated for the Ising model in the classical papers of Kac, Ward [10, and Vdovichenko [1]. ( See also 27, built upon [10, 11] and describing how to use the Kac, Ward-Vdovichenko method to solve the dimer model.) In contrary with the case of the embedded orbits, which play a key role in our manuscript, the number of immersed orbits is infinite even on a finite graph (since the length of the immersed orbits is not restricted), and thus the full partition function for a finite graph (which is also equal to a finite determinant) is represented in terms of an infinite product (not sum!) over the equivalence classes of the walks over the cyclic permutations. In this context the language of the Grassman/Fermion models extensively used in our mansucript is more appropriate for the finite graphical objects, i.e. embedded rather than more general immersed orbits, because of the nilpotent feature of the Grassmann variables.

We would also like to note that in this manuscript we did not give exhaustive description to the full class of det-easy problems on a given planar graph but described its rather broad, but still incomplete, subset. This incompleteness is in fact illustrated on a "counter-example" of the so-called \#X-matching model of Valiant [1-3], which is reducible to a det-easy model, however, via a set of more general (extended alphabet) gauge transformations, rather than binary gauge transformations discussed in our manuscript.

The manuscript is organized as follows. Section II starts with a brief description of the key graphical objects used through out the manuscript and then it is split into three Subsections. Sections IIA and IIB define the Wick Binary Model (WBG) model and the Grassmann Gaussian Graphical $\left(\mathrm{G}^{3}\right)$ model respectively, while Section IIC states the main result of the manuscript concerning relation between the two models under the proper, so-called suitable, choice of orientations entering definition of the $\mathrm{G}^{3}$ model. The respective Theorem is proved in Section III in a combinatorial way, while discussion of alternative topological considerations is given in Appendix A. Section IV is devoted to constructing efficiently a suitable orientation for a given planar graph. The construction of a suitable orientation is split into two steps. First step, discussed in Section IVA, describes a procedure of building an extended graph, defining a Kasteleyn orientation on it and then generating an induced orientation on the original graph. On the second step, described in Section IVB, we show that the induced orientation is a suitable orientation, thus resulting overall in a simple algorithm for evaluating the partition function of any WBG model on the planar graph. Section V discusses some details and examples of the gauge transformations, which transforms a WBG model to other det-easy models. The Section is broken in four Subsections. Section $\sqrt{\mathrm{VA}}$, based on materials from [4] review of the gauge transformation procedure, as well as examples that illustrate how the gauge transformations reduce the dimer model, ice model and Ising model on a planar graph to the general WBG models, given in Sections VB|VC|VD respectively. The aforementioned "counter-example", which is det-easy yet requires a more general reduction procedure, is discussed in Section $\mathrm{VE}$. An alternative generalization of the gauge transformation approach is discussed in Appendix B. Section VI concludes the manuscript with a brief summary and a discussion of future challenges.

\section{KEY OBJECTS, MODELS AND STATEMENTS}

We start describing briefly some common definitions used in this Section but also through out the paper.

Consider a finite connected non-oriented graph, $\mathcal{G}=\left(\mathcal{G}_{0}, \mathcal{G}_{1}\right)$, with $\mathcal{G}_{0}$ and $\mathcal{G}_{1} \subset\left\{\alpha \in 2^{\mathcal{G}_{0}} \mid \operatorname{card}(\alpha)=2\right\}$ being the (finite) sets of graph nodes/vertices and (undirected) edges/links, respectively. For $a, b \in \mathcal{G}_{0}$ we will often use a notation $a \sim b$ to describe the relation $\{a, b\} \in \mathcal{G}_{1}$, i.e., " $a$ and $b$ are connected by an edge". A directed edge (that goes from node $a \in \mathcal{G}_{0}$ to node $\left.b \in \mathcal{G}_{0}\right)$ is an ordered pair $(a, b) \in \mathcal{G}_{0} \times \mathcal{G}_{0}$ with $\{a, b\} \in \mathcal{G}_{1}$. There are exactly two directed edges $(a, b)$ and $(b, a)$ that represent (are associated with) a non-directed edge $\{a, b\} \in \mathcal{G}_{1}$. A triplet $a \rightarrow b \rightarrow c$ is an ordered set $(a, b, c) \in \mathcal{G}_{0} \times \mathcal{G}_{0} \times \mathcal{G}_{0}$ with $\{a, b\},\{b, c\} \in \mathcal{G}_{1}$ and $a \neq c$. With a minimal abuse we will use a convenient notation $(a, b) \in \mathcal{G}_{1}$ or even $(a, b) \in \mathcal{G}$ to describe the relation $\{a, b\} \in \mathcal{G}_{1}$, and $(a \rightarrow b \rightarrow c) \in \mathcal{G}_{1}$ or $(a \rightarrow b \rightarrow c) \in \mathcal{G}$ to describe the relation " $a \rightarrow b \rightarrow c$ is a triplet in $\mathcal{G}$ ".

All graphs discussed in this manuscript are planar, i.e., they can be drawn on the plane in such a way that their edges intersect only at the endpoints.

Let $\delta_{a}(\mathcal{G})$ be the set of vertices which are neighbors of vertex $a$ over graph $\mathcal{G}$, and $\left|\delta_{a}(\mathcal{G})\right|$ be the cardinality of the set, which we will also call degree/valence of $a$ in $\mathcal{G}$. The graph degree/valence of $\mathcal{G}$ is the maximal $\left|\delta_{a}(\mathcal{G})\right|$ among all the vertices of the graph. A graph is called homogeneous if all its vertices have the same valence. A 


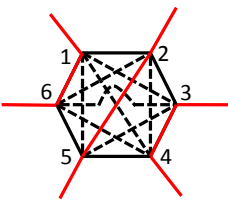

(a)

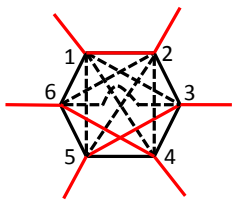

(b)

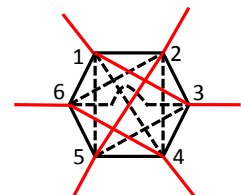

(c)

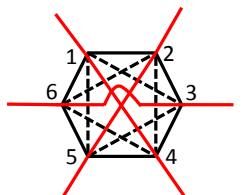

(d)

FIG. 1: Illustration of the Wick model construction. Four graph illustrates four representative terms (of the total of $(2 n-1)$ !!) for different 6-vertex contributions into Eq. 33, correspondent to (a) $W_{\{[1,6],[2,5],[3,4]\}}=W_{16} W_{25} W_{34}$ [zero crossing], (b) $W_{\{[1,2],[3,5],[4,6]\}}=-W_{12} W_{35} W_{46}$ [one crossing], (c) $W_{\{[1,3],[2,5],[4,6]\}}=W_{13} W_{25} W_{46}$ [two crossings], and (d) $W_{\{[1,4],[2,5],[3,6]\}}=$ $-W_{14} W_{25} W_{36}$ [three crossings], where the upper index of the 6-vertex is dropped to simplify the notations.

connected graph $\mathcal{G}$ is called irreducible if after withdrawing any single edge the graph stays connected; it is called reducible, otherwise. Starting with a reducible graph and withdrawing one-by-one the edges that increase the number of connected components we arrive at a disjoint union of irreducible graphs, referred to as the irreducible components of $\mathcal{G}$. The edges withdrawn in the above procedure are referred to as the connecting edges. Note that if we contract the irreducible components of $\mathcal{G}$ to points, the resulting graph will become a tree, whose edges are represented by the connecting edges of $\mathcal{G}$. Also note that an irreducible graph does not have vertices of degree one. A linear graph $\mathcal{L}$ of length $l$ is a graph with the set of vertices and edges being $\mathcal{L}_{0}=\left\{a_{0}, \ldots, a_{l}\right\}$ and $\mathcal{L}_{1}=\left\{\left\{a_{0}, a_{1}\right\}, \ldots,\left\{a_{l-1}, a_{l}\right\}\right\}$ respectively. A circular graph $\mathcal{S}$ of length $l$ has the following set of vertices and edges: $\mathcal{S}_{0}=\left\{a_{1}, \ldots, a_{l}\right\}$ and $\mathcal{S}_{1}=\left\{\left\{a_{1}, a_{2}\right\}, \ldots,\left\{a_{l-1}, a_{l}\right\},\left\{a_{l}, a_{1}\right\}\right\}$. For an irreducible graph $\mathcal{G}$ of degree three or higher a linear segment is a linear subgraph $\mathcal{L} \subset \mathcal{G}$ with the degree of vertices (in $\mathcal{G}) a_{1}, \ldots, a_{l-1}$ being two, and degree of $a_{0}, a_{l}$ exceeding two. For a circular segment the vertices $a_{2}, \ldots, a_{l}$ have degree two, whereas the degree of $a_{1}$ exceeds two. All irreducible graphs of degree two are circular. An irreducible graph of degree three does not have circular segments.

The layout of the the material in the remainder of the Section is as follows. The Wick Binary Graphical (WBG) model on a planar graph and the auxiliary objects, e.g. even generalized loops and intersection index, required for the model definition are introduced in Section II A. Then in Section II B we proceed to defining the Grassmann Gaussian Graphical $\left(\mathrm{G}^{3}\right)$ model and respective auxiliary graph objects, the complete (combined edge- and triplet-) orientation. Finally, in Section II C we define the notion of suitable complete orientation and state the main result of the manuscript describing relation between the WBG and the $\mathrm{G}^{3}$ models defined on the same graph.

\section{A. Wick Binary Graphical Model}

Consider a graph of an arbitrary degree and introduce the following notations

Definition II.1 (Generalized loop. Even Generalized loop $=\mathbb{Z}_{2}$-cycle.) Generalized loop of $\mathcal{G}$ is a subgraph of $\mathcal{G}$ which does not contain vertices of degree one (an empty set is also a generalized loop). Even generalized loop, or $\mathbb{Z}_{2}$-cycle, is a subgraph with all vertices of even degree.

Remark(s): Obviously, a simple cycle of $\mathcal{G}$ is also a generalized loop of $\mathcal{G}$. The term $\mathbb{Z}_{2}$-cycle establishes a connection with a language common in algebraic topology. A $\mathbb{Z}_{2}$-chain, $c \in \mathcal{C}_{1}\left(\mathcal{G}_{\mathbb{Z}_{2}}\right)$, is defined by a set of $\mathbb{Z}_{2}$-numbers $c=$ $\left\{c_{a b}=c_{b a}=0,1 \mid\{a, b\} \in \mathcal{G}_{1}\right\}$. We further introduce the standard boundary operator with its value at a vertex $a \in \mathcal{G}_{0}: \partial(\boldsymbol{c})_{a}=\sum_{b \sim a} c_{a b}(\bmod 2)$. A chain $c$ is called a $\mathbb{Z}_{2}$-cycle, if it has no boundary $\partial(\boldsymbol{c})=0$, i.e., $\forall a \in \mathcal{G}_{0}$ : $\partial(\boldsymbol{c})_{a}=0$. Even generalized loop, as a subgraph of $\mathcal{G}$ associated with a $\mathbb{Z}_{2}$ cycle $\gamma$, is constructed of edges with nonzero contributions, $\gamma_{a b}=1$. Therefore, in what follows we may think of $\gamma$ as either a $\mathbb{Z}_{2}$ structure on the graph or as of respective subgraph.

We will also need the following graph/vertex-local notations.

Local partitions and intersection index. For a finite set $A$ with even number card $(A)=2 k$ of elements let $P(A)$ be the set of all partitions of $A$ in $k$ distinct non-ordered pairs. An element $\xi \in P(A)$ can be viewed as a set of $k$ elements, represented by pairs $\left\{a, a^{\prime}\right\} \in \xi$ (with $a, a^{\prime} \in A$ ) that form the partition. We further note that, since our graph $\mathcal{G}$ is planar, each vertex $b$ is equipped with a cyclic ordering (we choose counterclockwise) of its adjacent vertices (neighbors) $a \sim b$. For a vertex $b$ we can place its neighbors $a \sim b$ on a circle $S^{1} \in \mathbb{R}^{2}$ standardly embedded into a plane, according to their cyclic ordering, and denoting by $I_{\alpha}=I_{\{a, c\}}$ a segment of a straight line 
that connects $a$ and $c$ we introduce the modulo-2 intersection index $I_{\alpha} \cdot I_{\alpha^{\prime}} \in \mathbb{Z}_{2}$, associated with two distinct pairs $\alpha=\{a, c\}$ and $\alpha^{\prime}=\left\{a^{\prime}, c^{\prime}\right\}$, as the number of intersections of $I_{\alpha}$ with $I_{\alpha^{\prime}}$. Obviously we can associate with a partition $\xi \in P\left(\left\{a_{1}, \ldots, a_{2 k}\right\}\right)$ of an even subset of neighbors of $b$ a $\mathbb{Z}_{2}$ intersection index by

$$
N_{b}\left(\left\{a_{1}, \ldots, a_{2 k}\right\} ; \xi\right)=\sum_{\left\{\alpha, \alpha^{\prime}\right\} \in 2^{\xi}}^{\alpha \neq \alpha^{\prime}} I_{\alpha} \cdot I_{\alpha^{\prime}}(\bmod 2)
$$

We are now ready to define one of our basic models. A binary statistical model on the graph is defined in terms of its partition function, describing a weighted sum over all allowed configurations.

WBG model. The partition function of the Wick Binary Graphical (WBG) model, dependent on the vector of weights $\boldsymbol{W}$, is given by

$$
\begin{aligned}
& Z_{W B G}(\boldsymbol{W})=\sum_{\gamma=\left\{\gamma_{a b}\right\} \in \mathcal{Z}_{1}\left(\mathcal{G} ; \mathbb{Z}_{2}\right)} \prod_{b \in \mathcal{G}_{0}}^{\exists a \sim b \mid \gamma_{a b} \neq 0} W_{\left\{a_{1}, \cdots, a_{2 k}\right\} \equiv\left\{a \mid a \sim b ; \gamma_{a b}=1\right\}}^{(b)}, \\
& W_{\left\{a_{1}, \cdots, a_{2 k}\right\}}^{(b)} \equiv \sum_{\xi \in P\left(\left\{a_{1}, \ldots, a_{2 k}\right\}\right)} W_{\xi, a_{1} \cdots a_{2 k}}^{(b)}, \quad W_{\xi, a_{1} \cdots a_{2 k}}^{(b)} \equiv(-1)^{N_{b}\left(\left\{a_{1}, \ldots, a_{2 k}\right\} ; \xi\right)} \prod_{\alpha \in \xi} W_{\alpha}^{(b)},
\end{aligned}
$$

where in the first line the summation goes over all $\mathbb{Z}_{2}$-cycles, i.e. even generalized loops (contributions associated with all odd generalized loops are assumed zero), of $\mathcal{G}$, and subsequent product is over all vertices of nonzero degree contributing the even generalized loop. The second line in Eq. (3) represents explicit expression of the higher vertex weight with $k \geq 2$ in terms of the lowest vertex weights $W_{a_{1} a_{2}}^{(b)}$, where the latter represents the case $k=1$.

$\operatorname{Remark(s):~(a)~Due~to~symmetric~nature~of~the~weights~we~can~write~} W_{\alpha}^{(b)}=W_{\left\{a_{i}, a_{j}\right\}}^{(b)}=W_{a_{i} a_{j}}^{(b)}=W_{a_{j} a_{i}}^{(b)}$ for $\alpha=\left\{a_{i}, a_{j}\right\}$.

(b) Overall, the expressions given by Eq. $\sqrt{3}$ can be interpreted as follows. Each contribution $W_{\xi, a_{1} \ldots a_{2 k}}^{(b)}$ to the sum is determined by a partition of the set $\left\{a_{1}, \ldots a_{2 k}\right\}$ into distinct pairs $\alpha_{1}, \ldots \alpha_{k}$. The contribution is given by the product $W_{\alpha_{1}}^{(b)} \ldots W_{\alpha_{k}}^{(b)}$ with the overall sign in front to be plus or minus, depending whether the total number of intersections between the segments $I_{\alpha_{i}}$ is even or odd. To make the construction, required to define the Wick model, transparent we illustrate it for $\left|\delta_{\gamma}(a)\right| / 2=3$ in Fig. 1 .

(c) We call the model of (2)3 Wick model to emphasize relation to the Wick theorem/rules used in the quantum field theory to express high-order correlation function via second-moments (covariances) in the case of Gaussian statistics. More specifically, the $(2 k)$-th order contribution $W_{\left\{a_{1}, \cdots, a_{2 k}\right\}}^{(b)}$ to the weight function is given by the Pfaffian of a $(2 k) \times(2 k)$ skew-symmetric matrix, with the matrix elements given by the binary weights $W_{a_{i} a_{j}}^{(b)}$, that can be viewed as a Gaussian integral over Grassman variables.

(d) The introduction of the set of constraints given by Eq. (3) is motivated by our desire to establish term-by-term relation between the yet to be defined $\mathrm{G}^{3}$ model and the discrete model (2).

\section{B. Orientations and the Gaussian Grassmann Graphical Model}

As in the preceding Subsection we will start introducing key objects (orientations) required to define the $\mathrm{G}^{3}$ model.

Definition II.2 (Edge-orientation.) A graph edge-orientation $\boldsymbol{\sigma}$ is a particular choice of a directed representative $(a, b)$ for any undirected edge $\{a, b\} \in \mathcal{G}_{1}$, or equivalently a set of numbers $\sigma_{a b}= \pm 1$ associated with directed edges $(a, b)$ with the skew-symmetric condition $\sigma_{a b}=-\sigma_{b a}$.

Definition II.3 (Triplet-orientation. Complete orientation.) A graph triplet-orientation $\boldsymbol{\varsigma}$ is a set of numbers $\varsigma_{a c}^{(b)}= \pm 1$, associated with the triplets $a \rightarrow b \rightarrow c$ with $\varsigma_{a c}^{(b)}=-\varsigma_{c a}^{(b)}$. A set $(\boldsymbol{\sigma}, \boldsymbol{\varsigma})$, with $\boldsymbol{\sigma}$ being a graph edge orientation (see Definition II.2) is called a complete orientation ${ }^{3}$.

\footnotetext{
${ }^{3}$ In the following we will use the term orientation to describe complete orientation.
} 
Remark(s): Note that there are no triplets $a \rightarrow b \rightarrow c$, associated with the nodes $b \in \mathcal{G}_{0}$ of valence (degree) one. Therefore, the triplet-orientation components $\varsigma_{a c}^{(b)}$, associated with the nodes $b$ of degree one are represented by an empty set.

$\mathbf{G}^{3}$ model. The partition function of the $\mathrm{G}^{3}$ model, dependent on a complete orientation of the graph, $(\boldsymbol{\sigma}, \boldsymbol{\varsigma})$, and also dependent on the vector of weights $\boldsymbol{W}$, is defined in terms of the following integral (sum) over the Grassmann anti-commuting, variables $\varphi$ :

$$
Z_{\mathrm{G}^{3}}(\boldsymbol{\sigma}, \boldsymbol{\varsigma} ; \boldsymbol{W})=\frac{\int \exp \left(\frac{1}{2} \sum_{(b \rightarrow a \rightarrow c) \in \mathcal{G}_{1}} \varphi_{a b} \varsigma_{b c}^{(a)} W_{b c}^{(a)} \varphi_{a c}\right) \exp \left(\frac{1}{2} \sum_{(a, b) \in \mathcal{G}_{1}} \varphi_{a b} \sigma_{a b} \varphi_{b a}\right) \prod_{(a, b)} d \varphi_{a b}}{\int \exp \left(\frac{1}{2} \sum_{(a, b) \in \mathcal{G}_{1}} \varphi_{a b} \sigma_{a b} \varphi_{b a}\right) \prod_{(a, b)} d \varphi_{a b}},
$$

where $\forall(a, b),(c, d) \in \mathcal{G}_{1} \quad \varphi_{a b} \varphi_{c d}=-\varphi_{c d} \varphi_{a b}$. There are two independent Grassmann variables per edge (one per directed edge), and the Berezin/Grassman integrals in Eq. (4) are defined according to standard Berezin rules, $\forall(a, b) \in \mathcal{G}_{1}: \quad \int d \varphi_{a b}=0, \quad \int \varphi_{a b} d \varphi_{a b}=1$.

Remark(s): (a) The partition function of the $\mathrm{G}^{3}$ model can also be interpreted as a result of the following Gaussian statistical average (expectation value) over the Grassmann variables

$$
Z_{\mathrm{G}^{3}}(\boldsymbol{\sigma}, \boldsymbol{\varsigma} ; \boldsymbol{W})=\left\langle\exp \left(\frac{1}{2} \sum_{(b \rightarrow a \rightarrow c) \in \mathcal{G}_{1}} \varphi_{a b} \varsigma_{b c}^{(a)} W_{b c}^{(a)} \varphi_{a c}\right)\right\rangle .
$$

(b) The denominator in Eq. (4), which is \pm 1 by construction, is introduced for convenience to enforce the normalization condition, $Z_{\mathrm{G}^{3}}(\boldsymbol{\varsigma} ; \boldsymbol{\sigma} ; \mathbf{0})=1$.

(c) Significance of the $\mathrm{G}^{3}$ model, as of any other Gaussian Grassman model, is in the fact that its partition/generating function is a Pfaffian. Indeed, the numerator in Eq. (4) is the Pfaffian of the skew-symmetric matrix (of the size twice the number of edges in the graph) with the following elements

$$
H_{i j}=\left\{\begin{array}{c}
\varsigma_{b c}^{(a)} W_{b c}^{(a)}, i=(a, b) \& j=(a, c), \text { where } b \neq c \sim a \\
\sigma_{a b}, \quad i=(a, b), \& j=(b, a) .
\end{array}\right.
$$

Thus calculating the partition function of the $\mathrm{G}^{3}$ model is easy, as requiring at most $O\left(\left|\mathcal{G}_{1}\right|^{3}\right)$ operations.

(d) When the graph $\mathcal{G}$ is a tree, the numerator in Eq. (4) does not depend on $\boldsymbol{W}$, and therefore the partition function $Z_{\mathrm{G}^{3}}(\boldsymbol{\sigma}, \boldsymbol{\varsigma} ; \boldsymbol{W})=1$ is trivial. Nontrivial dependence of the partition function on $\boldsymbol{W}$ appears due to the loops in the graph, as will be discussed in details later in the manuscript.

\section{Equivalence between the WBG model and the $\mathrm{G}^{3}$ model}

The main subject of this Subsection (and also of the manuscript) will consist in establishing equivalence between the WBG model and the $\mathrm{G}^{3}$ model for a given (arbitrary) set of edge-weights, $\boldsymbol{W}$. However, the partition function of the former model depends of $\boldsymbol{W}$ only, while the partition function of the later model depends on both the edge-weight vector $\boldsymbol{W}$ and on a complete orientation $(\boldsymbol{\sigma}, \boldsymbol{\varsigma})$ of the graph. Therefore, the aforementioned equivalence between the models is established only for some special choice(s) of the complete orientation which is detailed in some number of forthcoming definitions preceding the main theorem/statement.

Definition II.4 (Closed, immersed and embedded walk. Immersed and embedded orbit. Oriented edges and triplets of the walk/orbit.) A closed walk $C$ of length $l(C)$ on a graph $\mathcal{G}$ (not necessarily planar) is an ordered set $C=\left(c_{0}, \ldots, c_{n}\right)$ of nodes $c_{j} \in \mathcal{G}_{0}$, such that $c_{j}$ and $c_{j+1}$ are adjacent for all $j=0, \cdots, n-1$, and $c_{n}=c_{0} . A$ closed walk is called immersed if $c_{j-1} \neq c_{j+1}, \forall j=1, \ldots, n$, i.e., the walk does not involve backtracking events (hereafter we use a natural agreement $\left.c_{n+1}=c_{1}\right)$. A closed walk is called embedded if $c_{j} \neq c_{k}, \forall j \neq k$, i.e., the path does not have self-intersections. An equivalence class of closed walks with respect to the cyclic permutations of the path nodes is called an orbit. An immersed orbit and an embedded orbit is an equivalence class, represented by an immersed closed walk and an embedded closed walk, respectively. The ordered pairs $\left(c_{j-1}, c_{j}\right)$ and triplets $\left(c_{j-1}, c_{j}, c_{j+1}\right)$ for $j=1, \ldots, n$ are called the oriented edges and triplets of $C$, respectively. 
Remark(s): Obviously any embedded closed walk (or orbit) is immersed. The definition (II.4) assumes that orbits are oriented, however we will also be discussing undirected orbits as equivalence classes of orbits with respect to orientation change, sometimes without additional clarification (when it does not cause a confusion). An undirected embedded orbit is synonymous to a simple cycle. The terms immersed and embedded are borrowed from topology and are used here based on the fact that immersed and embedded closed walks can be viewed as discrete counterparts of the loops, immersed and embedded, respectively into a plane. An immersed loop into a plane, or equivalently an immersion $S^{1} \rightarrow \mathbb{R}^{2}$ of a circle into a plane is a smooth closed trajectory with everywhere non-zero velocity. This analogy is detailed in Appendix A and applied to unveil the topology that stands behind the equivalence of the WBG and $\mathrm{G}^{3}$ models.

Sign of an immersed orbit, associated with a given complete orientation. Given a complete graph orientation $(\boldsymbol{\sigma}, \boldsymbol{\varsigma})$, we associate a $\operatorname{sign} \varepsilon(C)=\varepsilon_{\boldsymbol{\sigma}, \boldsymbol{\varsigma}}(C)= \pm 1$ with any immersed orbit $C$ by

$$
\varepsilon(C)=\left(\prod_{(b \rightarrow a \rightarrow c) \in C} \varsigma_{b c}^{(a)}\right) \prod_{(a, b) \in C} \sigma_{a b}=\prod_{j=0}^{l(C)-1} \varsigma_{c_{j-1} c_{j+1}}^{\left(c_{j}\right)} \prod_{j=0}^{l(C)-1} \sigma_{c_{j} c_{j+1}} .
$$

Remark(s): Note that the binary function $\varepsilon$ is obviously invariant with respect to cyclic permutations of $C$, therefore, the signs $\varepsilon(C)$ are defined for oriented immersed orbits. A change of orientation changes the sign of each factor in the products in the rhs of Eq. (6), and since the number of the $\sigma$-factors is the same as the number of $\varsigma$-factors, $\varepsilon(C)$ is invariant with respect to the orientation changes. Therefore, $\varepsilon$ is actually correctly defined for a non-oriented immersed orbit as well.

Decomposition of a cycle. A generic $\mathbb{Z}_{2}$-cycle can be decomposed into a set of immersed orbits in a variety of ways. It is easy to realize that each decomposition can be labeled by a set of variables $\boldsymbol{\xi}=\left\{\xi_{a}\right\}_{a \in \mathcal{G}_{0}}$, referred to as partition data where $\xi_{a}$ for $a \in \gamma$ denotes a partition of the set of the adjacent edges $\{a, b\} \in \gamma$ (or equivalently adjacent nodes $b \in \gamma$ ) into distinct non-ordered pairs. Formally $\xi_{a}$ can be viewed as a set whose elements are given by the aforementioned distinct pairs. The partition data $\boldsymbol{\xi}$ naturally provides a set $\left\{C_{1}(\gamma, \boldsymbol{\xi}), \ldots, C_{n(\gamma, \boldsymbol{\xi})}(\gamma, \boldsymbol{\xi})\right\}$ of immersed orbits that decompose $\gamma$. The partition variables $\xi_{a}$ have been already used earlier to express the higher vertex weights of a Wick binary model in terms of the lowest vertex weights, see Eqs. (2) and (3).

Definition II.5 (Decomposed cycle. Decomposition Components. Total self-intersection index of a decomposed cycle.) A pair $(\gamma, \boldsymbol{\xi})$, where $\gamma$ is a $\mathbb{Z}_{2}$-cycle and $\boldsymbol{\xi}$ is the partition data, associated with its vertices is called a decomposed cycle. The set $\left\{C_{1}(\gamma, \boldsymbol{\xi}), \ldots, C_{n(\gamma, \boldsymbol{\xi})}(\gamma, \boldsymbol{\xi})\right\}$ is called a decomposition of $\gamma$ associated with $\boldsymbol{\xi}$. The immersed orbits $C_{j}(\gamma, \boldsymbol{\xi})$ are referred to as the decomposition components. The total self-intersection index of a decomposed cycle $N(\gamma, \boldsymbol{\xi}) \in \mathbb{Z}_{2}$ is defined as a sum $N(\gamma, \boldsymbol{\xi})=\sum_{a \in \gamma} N_{a}(\gamma, \boldsymbol{\xi})=\sum_{a \in \gamma} \sum_{\left\{\alpha, \alpha^{\prime}\right\}}^{\alpha \neq \alpha_{a}^{\prime}} I_{\alpha} \cdot I_{\alpha^{\prime}}$ over the cycle vertices, where the (elementary) intersection index $I_{\alpha} \cdot I_{\alpha^{\prime}}$ was defined in Subsection II A.

Remark(s): (a) The definition of the local intersection index $N_{a}(\gamma, \boldsymbol{\xi})$ is fully consistent with Eq. 11), since $N_{a}(\gamma, \boldsymbol{\xi})=$ $N_{a}\left(\gamma_{a}, \xi_{a}\right)$, where $\gamma_{a}$ is the set of neighbors of $a$ that belong to $\gamma$.

(b) Note that the decomposition components are immersed orbits of a special type: they do not have self-intersecting and intersecting edges, and all intersections and self-intersections (if any) occur at the vertices only. In particular, the components generate $\mathbb{Z}_{2}$-cycles that will be denoted in the same way, and the decomposition can be also represented as $\gamma=\sum_{j=1}^{n(\gamma, \boldsymbol{\xi})} C_{j}(\gamma, \boldsymbol{\xi})$.

The just introduced notion of a decomposed cycle allows a convenient definition of a suitable orientation.

Definition II.6 (Suitable Complete Orientation.) A (complete) orientation is called suitable if for any decomposed cycle $(\gamma, \boldsymbol{\xi})$ the following relation holds

$$
(-1)^{q(\gamma, \boldsymbol{\xi})}=1
$$

where the binary function $q(\gamma, \boldsymbol{\xi})$ is defined by

$$
(-1)^{q(\gamma, \boldsymbol{\xi})}=(-1)^{N(\gamma, \boldsymbol{\xi})} \prod_{j=1}^{n(\gamma, \boldsymbol{\xi})}\left(-\varepsilon\left(C_{j}(\gamma, \boldsymbol{\xi})\right)\right) .
$$

Note that, as discussed later in Section IV] such suitable complete orientations do exists.

We are now in the position to state the main theorem.

Theorem II.7 For any connected planar graph (of an arbitrary degree) the discrete-variable Wick model (2) and fermion model (4) are term-by-term, i.e. fully, equivalent to each other, i.e., $Z_{G^{3}}(\boldsymbol{\varsigma}, \boldsymbol{\sigma} ; \boldsymbol{W})=Z_{W B G}(\boldsymbol{W})$, for any $\boldsymbol{W}$, provided $(\boldsymbol{\sigma}, \boldsymbol{\varsigma})$ is a suitable orientation of $\mathcal{G}$. 


\section{PROOF OF THE WBG AND G ${ }^{3}$ EQUIVALENCE}

The main task of this Section is to sketch the proof of the Theorem II.7. We start by making the following (almost obvious) statement that reduces the proof of Theorem II.7 to the case of irreducible graphs $\mathcal{G}$.

Proposition III.1 (i) The partition functions $Z_{W B G}(\boldsymbol{W})$ and $Z_{G^{3}}(\boldsymbol{\varsigma}, \boldsymbol{\sigma} ; \boldsymbol{W})$ of the $W B G$ and $G^{3}$ models both satisfy the factorization property: the partition function for $\mathcal{G}$ is given by the product of the partition functions of the corresponding models restricted to the irreducible components of $\mathcal{G}$. (ii) An orientation $(\boldsymbol{\sigma}, \boldsymbol{\varsigma})$ on $\mathcal{G}$ is suitable if and only if its restriction to any irreducible component of $\mathcal{G}$ is suitable.

Proof: For the WBG model both the factorization property (i) and the feature (ii) follow immediately from the fact that any $\mathbb{Z}_{2}$-cycle on $\mathcal{G}$ is restricted to the disjoint union of its irreducible components. The factorization property for the $\mathrm{G}^{3}$ model is obtained by consecutive integrations of the numerator and denominator of Eq. (4) over the pairs $d \varphi_{a b} d \varphi_{b a}$ of Grassmann variables related to the connecting edges $\{a, b\}$. Each integration is performed by representing, $\exp \left(\varphi_{a b} \varphi_{b a} / 2\right)=1+\varphi_{a b} \varphi_{b a} / 2$. Substitution of the first term into the numerator/denominator of Eq. (4) results in a product of two Grassmann integrals of even functions over an odd number of integrations, which yields zero. Substitution of the second term, followed by the integration over $d \varphi_{a b} d \varphi_{b a}$ results in the product of two integrals over two unconnected graphs obtained by eliminating the connecting edge $\{a, b\}$.

Now we are in the position to describe a

Proof of Theorem II.7; Substituting Eq. (3), which expresses the higher order vertex functions in terms of the respective edge terms, into Eq. 22, we obtain the following expression for the partition function in the form of a sum over the combined variables $(\gamma, \boldsymbol{\xi})$

$$
Z_{\mathrm{WBG}}(\boldsymbol{W})=\sum_{(\gamma, \boldsymbol{\xi})}(-1)^{N(\gamma, \boldsymbol{\xi})} \prod_{j=1}^{n(\gamma, \boldsymbol{\xi})} r\left(C_{j}(\gamma, \boldsymbol{\xi})\right), \quad r(C)=\prod_{k=1}^{l(C)} W_{c_{k-1} c_{k+1}}^{\left(c_{k}\right)} .
$$

Eq. (9) is derived by means of re-grouping the factors correspondent to the lowest weights $W_{a c}^{(b)}$ and keeping together terms correspond to the same immersed orbits $C_{j}(\gamma, \boldsymbol{\xi})$ participating in the decomposition of $\gamma$ determined by $\boldsymbol{\xi}$. The sign factor in Eq. (9) is given by the product of the respective signs associated with the nodes of $\gamma$, and it is thus determined by the total number of intersections, $N(\gamma, \boldsymbol{\xi})$, introduced in Definition (II.5).

A similar to Eq. (9) expansion for the partition function of the Fermion model is

$$
Z_{\mathrm{G}^{3}}(\boldsymbol{\varsigma}, \boldsymbol{\sigma} ; \boldsymbol{W})=\sum_{(\gamma, \boldsymbol{\xi})} \prod_{j=1}^{n(\gamma, \boldsymbol{\xi})}\left(-\varepsilon\left(C_{j}(\gamma, \boldsymbol{\xi})\right)\right) \prod_{j=1}^{n(\gamma, \boldsymbol{\xi})} r\left(C_{j}(\gamma, \boldsymbol{\xi})\right) .
$$

It can be rationalized as follows. We represent the exponential under the average in Eq. (4) as a product of the vertex exponentials (labeled by $a$ ) and expand the vertex exponentials in the natural bilinear combinations of the Grassmann variables $\varphi$. Each term of the expansion is naturally labeled by a set $\boldsymbol{\xi}$ of partition variables. We further compute the expectation value of each individual term in the expansion, using the Wick's theorem. Since the two-point correlation functions of the Grassmann variable is, $\left\langle\varphi_{a b} \varphi_{b a}\right\rangle=\sigma_{a b}$, when $\{a, b\} \in \mathcal{G}_{1}$ and, $\left\langle\varphi_{a b} \varphi_{b a}\right\rangle=0$, otherwise, the set $\boldsymbol{\xi}$ of the partition variables provides a non-zero contribution to the partition function if and only if it satisfies the following property: If $\{a, b\}$ participates in the local partition at node $a$, then $\{b, a\}=\{a, b\}$ participates at the local partition at node $b$. For a partition $\boldsymbol{\xi}$ that satisfies this property we can build the associated cycle $\gamma$ consisting of edges $\{a, b\}$ that participate in the local partitions. We further re-group the factors $\varphi_{a b} \varsigma_{b c}^{(a)} W_{b c}^{(a)} \varphi_{a c}$ by keeping together the terms that correspond to the same immersed orbits $C_{j}(\gamma, \boldsymbol{\xi})$ participating in the decomposition of $\gamma$. The decomposition is determined by $\boldsymbol{\xi}$, followed by evaluating the expectation values using the Wick's theorem, in particular with making use of the form of the two-point correlation functions. After re-grouping the factors correspondent to each immersed orbit, and combining together the $W_{a c}^{(b)}, \sigma_{a b}$ and $\varsigma_{a c}^{(b)}$ factors, this results in Eq. 10 .

By Definition II.6 the sign factors in all the individual contributions to the partition functions $Z_{\mathrm{WBG}}(\boldsymbol{W})$ and $Z_{\mathrm{G}^{3}}(\boldsymbol{\varsigma}, \boldsymbol{\sigma} ; \boldsymbol{W})$ (labeled by respective suitable orientation, $(\gamma, \boldsymbol{\xi})$ ) are the same, which proves the statement of the theorem.

\section{CONSTRUCTION OF A SUITABLE ORIENTATION}

In this Section we show how to construct efficiently a suitable orientation, required to compute the partition function of the WGB model efficiently, according to the Theorem II.7. The construction of the suitable orientation is split 


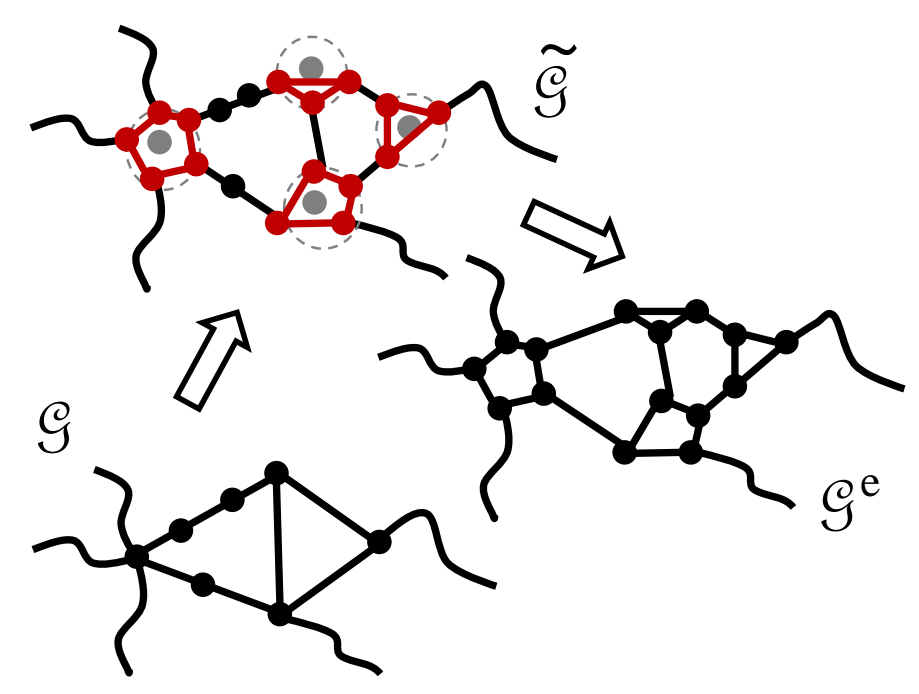

FIG. 2: Construction of the extended graph $\mathcal{G}^{e}$ associated with $\mathcal{G}$. A degree three graph $\tilde{\mathcal{G}}$ is constructed on an intermediate step. $\tilde{\mathcal{G}}$ is obtained from $\mathcal{G}$ by extending vertices. $\mathcal{G}^{e}$ is obtained from $\mathcal{G}$ by replacing linear segments with elementary edges.

into two steps. First step, discussed in Section IVA describes transformation of the original irreducible graph to the respective extended graph, $\mathcal{G}^{e}$, which is a homogeneous graph of degree three with even number of vertices. We also show in this Subsection how a Kasteleyn orientation of edges on the extended graph generates a so-called induced orientation on the original graph. Second step, undertaken in Section IVB, focuses on showing that an induced orientation of the original graph is always suitable, i.e. satisfies the condition of Theorem II.7.

Note that Proposition III.1 reduces the problem of identifying suitable orientations to the case when $\mathcal{G}$ is irreducible. Any irreducible graph of degree two is a cyclic graph, for which suitable orientations are identified in an obvious way. Therefore, throughout this section the graph $\mathcal{G}$, on which our models are defined is assumed irreducible and of degree three or higher (even when not explicitly stated), which does not lead to any loss of generality.

\section{A. Extended Graph and Induced Orientation}

Construction of the extended graph, $\mathcal{G}^{e}$, illustrated in Fig. (2), is done in two steps. We start from an original irreducible graph $\mathcal{G}$ of degree three or higher, which, in particular does not have vertices of degree one, and extend its vertices of degree three or higher to circles (or polygons) so that the adjacent edges of the original graph are connected to the polygon vertices. The procedure results in an irreducible graph $\tilde{\mathcal{G}}$ of degree three, which is however not necessarily homogeneous degree three, as it may have linear segments consisting of vertices of degree two. We further replace the linear segments of $\tilde{\mathcal{G}}$ with elementary edges to obtain an irreducible homogeneous graph $\mathcal{G}^{e}$ of degree three.

Definition IV.1 (Extended Graph. Extending Polygons.) The constructed above (in the preceding paragraph) irreducible homogeneous graph $\mathcal{G}^{e}$ of degree three is called the extended graph associated with an irreducible graph $\mathcal{G}$. The polygons that replace the vertices of degree three and higher are called the extending polygons.

We continue working the irreducible degree three homogeneous degree three graph $\mathcal{G}^{e}$ and observe that by construction it has an even number of vertices. Thus, following Kasteleyn [15, 16] (see also [26] for a comprehensive review) we construct the so-called Kasteleyn edge orientation on the graph. (See also Fig. 3 for an illustration.)

Definition IV.2 (Kasteleyn edge orientation.) An edge orientation of $\mathcal{G}^{e}$ (which is a planar graph with even number of vertices) is called Kasteleyn edge orientation of the graph if for any (even or odd) face of the graph (possibly under exception of the outer face) the number of clockwise-oriented (negative) edges is odd. This is formalized in terms of the vector, $\boldsymbol{\sigma}^{e}=\left(\sigma_{a b}^{e}= \pm 1 \mid\{a, b\} \in \mathcal{G}^{e}\right)$, where $\sigma_{a b}^{e}=+1$ if the arrow/orientation assigned to the undirected edge $\{a, b\}$ goes from a to $b$, and $\sigma_{a b}^{e}=-1$ otherwise.

Furthermore, $\mathcal{G}^{e}$ is also equipped with a natural triplet orientation, built using the following rule: we set $\varsigma_{b c}^{(a) ; e}=1$, if going from $b$ to $c$ represents a left most turn at $a$, and $\varsigma_{b c}^{(a) ; e}=-1$ otherwise. (See Fig. 3 b for an illustration.) $\varsigma$ is 


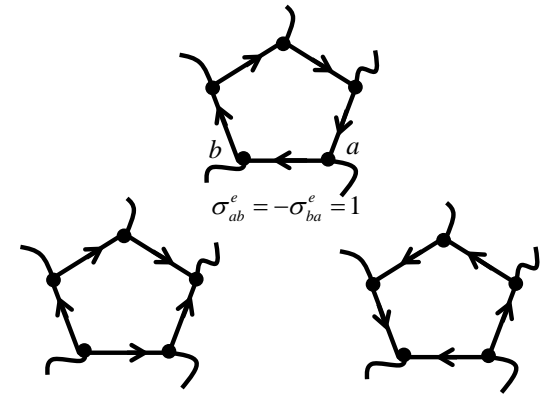

(a)

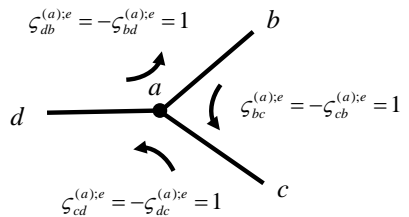

(b)

FIG. 3: Fig. (a) illustrates construction of the Kasteleyn orientation of edges - the three examples (with one, three and five clockwise edges per face respectively) are all legitimate Kasteleyn orientations of the selected internal face. Fig. (b) illustrates construction of a triplet orientation of a vertex of degree three (i.e. a vertex of $\mathcal{G}^{e}$ ).

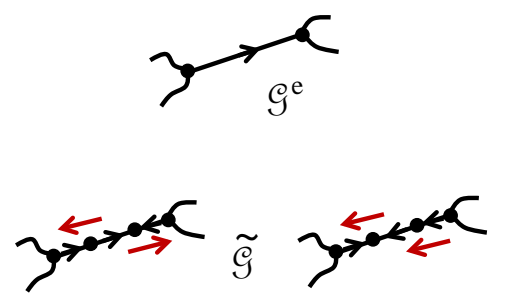

(a)

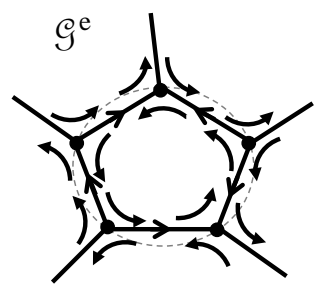

(b)
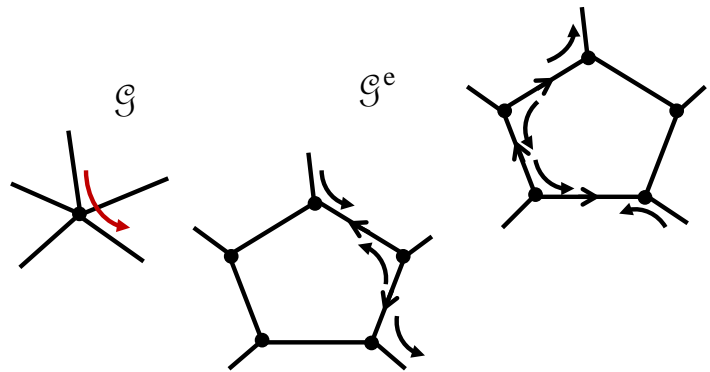

(c)

FIG. 4: Fig. (a) shows two examples of possible choice of edge and triplet orientations within a linear segment of $\tilde{\mathcal{G}}$. The two examples are compatible, i.e. they both results according to Eq. 111) in the same orientation of the respective single edge in $\mathcal{G}^{e}$ replacing the linear segment in $\tilde{\mathcal{G}}$. The number of arrows, marking both edge and triplet orientations within the linear segment of $\tilde{\mathcal{G}}$, directed against the resulting arrow/orientation of the respective edge of $\mathcal{G}^{e}$ is even. Fig. (b) shows fragment of complete Kasteleyn orientation on $\mathcal{G}^{e}$ and the related induced orientation of the respective triplet on $\mathcal{G}$. Fig. (c) illustrates how two equivalent ways of counting orientations on $\mathcal{G}^{e}$ results in the same triplet orientation on $\mathcal{G}$, correspondent to the triplet from Fig. (b): the total number of the upper/left orientation within either of the paths (shown on left and right figure) which are against the resulting (red) arrow on $\mathcal{G}$ is even.

a vector constructed from all the triplet orientations of $\mathcal{G}^{e}$. The apparently sloppy construction is actually rigorous, since a planar graph, in particular $\mathcal{G}^{e}$, is supplied with a cyclic (e.g., counterclockwise) ordering of edges, adjacent to any node.

Definition IV.3 (Kasteleyn (Complete) Orientation.) A constructed above triplet orientation $\boldsymbol{\varsigma}^{e}$ is called the left triplet orientation of $\mathcal{G}^{e} . A$ (complete) orientation $\left(\boldsymbol{\sigma}^{e}, \boldsymbol{\varsigma}^{e}\right)$ of an irreducible homogeneous graph $\mathcal{G}^{e}$ of degree three, represented by a Kasteleyn edge orientation $\boldsymbol{\sigma}^{e}$ and the left triplet orientation $\boldsymbol{\varsigma}$ is called a Kasteleyn (complete) orientation of $\mathcal{G}^{e}$.

Inducing orientations. Our next task becomes to generate from $\left(\boldsymbol{\sigma}^{e}, \boldsymbol{\varsigma}^{e}\right)$, defined on $\mathcal{G}^{e}$, a set of useful orientations $(\boldsymbol{\sigma}, \boldsymbol{\varsigma})$ on the original graph $\mathcal{G}$. This task is solved in a number of steps. We first build a set of orientations $(\tilde{\boldsymbol{\sigma}}, \tilde{\boldsymbol{\varsigma}})$ on $\tilde{\mathcal{G}}$ by staying with the left triplet orientation for vertices of degree three and choosing the edge and triplet orientations on the linear segments of $\tilde{\mathcal{G}}$ (end vertices excluded) in an arbitrary way with the only constraint per linear segment that the product,

$$
\tilde{\sigma}_{a_{0} a_{1}} \ldots \tilde{\sigma}_{a_{l-1} a_{l}} \tilde{\varsigma}_{a_{0} a_{2}}^{\left(a_{1}\right)} \ldots \tilde{\varsigma}_{a_{l-2} a_{l}}^{\left(a_{l-1}\right)}=\sigma_{a_{0} a_{l}}^{e},
$$

of all the edge an triplet sign factors reproduce the sign factor for the corresponding edge of $\mathcal{G}^{e}$. See Fig. 4 4 a for illustration of the construction. Then, we turn to constructing orientation on $\mathcal{G}$, first building respective components of $(\boldsymbol{\sigma}, \boldsymbol{\varsigma})$ for degree two vertices to be identical to the respective (and just constructed) orientations of the degree two vertices on $\tilde{\mathcal{G}}$. The components of $\varsigma$ related to degree three and higher vertices of $\mathcal{G}$ are defined as the products of the 


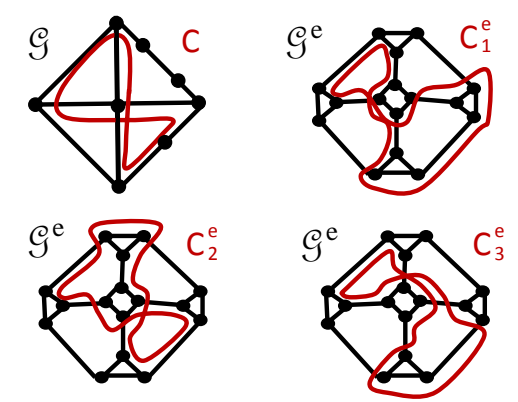

FIG. 5: Illustration of the lifting procedure. Top left: an example of $\mathcal{G}$ (black) and an immersed orbit $C$ (red). Three other sub-figures show three different (but equivalent in the sense of their respective $\varepsilon_{\boldsymbol{\sigma}^{e}, \boldsymbol{\varsigma}^{e}}\left(C^{e}\right)$ contributions, see Proposition IV.5) lifted version of $C$ on the respective extended graph, $\mathcal{G}^{e}$.

edge and inner triplet orientation factors of $\boldsymbol{\sigma}^{e} \boldsymbol{\varsigma}^{e}$, respectively, over the paths that connect the corresponding edges going over the extending polygons in either (counterclockwise or clockwise) direction, as shown in Fig. 4 p,c. The Kasteleyn nature of the complete orientation $\left(\sigma^{e}, \boldsymbol{\varsigma}^{e}\right)$ ensures the independence of $\boldsymbol{\varsigma}$ on the choice of the directions of the connecting paths.

Definition IV.4 (Induced Orientation.) A (complete) orientation $(\boldsymbol{\sigma}, \boldsymbol{\varsigma})$ of an irreducible graph, constructed above (in the preceding paragraph) is called an induced (complete) orientation, or more specifically an orientation induced from a Kasteleyn edge orientation $\boldsymbol{\sigma}^{e}$ of $\mathcal{G}^{e}$.

Lifting immersed orbits to the extended graph. Induced orientations are constructed in a way that the $\varepsilon(C)$-factors for the immersed orbits in $\mathcal{G}$, defined by Eq. (6), can be studied by lifting them to the extended graph. Given an immersed orbit in $\mathcal{G}$ we first lift it to $\tilde{\mathcal{G}}$ by connecting the ends of the linear segments of $C$ with paths going over the extending polygons. By further replacing the linear segments with elementary edges we obtain a lifted orbit in $\mathcal{G}^{e}$. Obviously, by construction a lifted orbit, $C^{e}$, is not unique and is specified by the choice of the directions (counterclockwise/clockwise) of the connecting paths. Lifting procedure is illustrated in Fig. 5 .

Proposition IV.5 Let $(\boldsymbol{\sigma}, \boldsymbol{\varsigma})$ on $\mathcal{G}$ be induced from a Kasteleyn orientation $\left(\boldsymbol{\sigma}^{e}, \boldsymbol{\varsigma}^{e}\right)$ on $\mathcal{G}^{e}$, and $C^{e}$ be any lift of an immersed orbit $C$ of $\mathcal{G}$ to the extended graph $\mathcal{G}^{e}$. Then $\varepsilon_{\boldsymbol{\sigma}^{e}, \boldsymbol{\varsigma}^{e}}\left(C^{e}\right)=\varepsilon_{\boldsymbol{\sigma}, \boldsymbol{\varsigma}}(C)$.

Proof: The statement follows directly from the construction of the induced orientation. For $\varepsilon_{\boldsymbol{\sigma}, \boldsymbol{\varsigma}}(C)$ the products of edge an inner triplet factors over the linear segments reproduce the edge factors $\boldsymbol{\sigma}^{e}$ of the corresponding edges of $\mathcal{G}^{e}$. The triplet factors of $\varsigma$ for vertices of degree three or higher reproduce the products of the edge and inner triplet factors of $\left(\boldsymbol{\sigma}^{e}, \boldsymbol{\varsigma}^{e}\right)$ over the connecting paths. The triplets that connect the linear segments to the connecting paths always come in pairs with two right/left turns for the counterclockwise/clockwise choice of the connecting path.

The important role of the Kasteleyn complete orientations and the corresponding induced complete orientations is described by the following statements.

Proposition IV.6 A Kasteleyn (complete) orientation of an irreducible homogeneous planar graph of degree three, $\mathcal{G}^{e}$ is suitable. According to Definition II.6, this is obviously equivalent to the statement that $\varepsilon\left(C^{e}\right)=-1$ for any embedded curve (simple cycle) $C^{e}{ }^{4}$.

Proof: The simple cycle $C^{e}$ partitions the plane into two connected regions, interior and exterior respectively. Therefore, $C^{e}=\partial \mathcal{M}$ is the boundary of the "inside" connected component (the one whose closure is compact), represented by a planar graph $\mathcal{M} \subset \mathcal{G}^{e}$ (the boundary is included). It is enough to prove the statement for the counterclockwise orientation of $C^{e}$, throughout this proof all involved orientations are counterclockwise. We further represent $\varepsilon\left(C^{e}\right)=\varepsilon_{\boldsymbol{\sigma}^{e}} \varepsilon_{\boldsymbol{s}}$ as a product of edge and triplet contributions along the embedded curve $C^{e}$, and denote by $n_{0}, n_{1}, n_{2}$ and $l$ the number of vertices, edges, and faces of $\mathcal{M}$ and the length (number of edges) of $C^{e}$, respectively. Considering

\footnotetext{
${ }^{4}$ We may consider any irreducible homogeneous graph of degree three here, and not necessarily the graph $\mathcal{G}^{e}$ extended from an arbitrary irreducible planar graph. However, since the proposition will be applied in the following solely to the extended graph, $\mathcal{G}^{e}$, we choose not to introduce new notations for an arbitrary irreducible homogeneous graph of degree three, using $\mathcal{G}^{e}$ for this purposes here.
} 


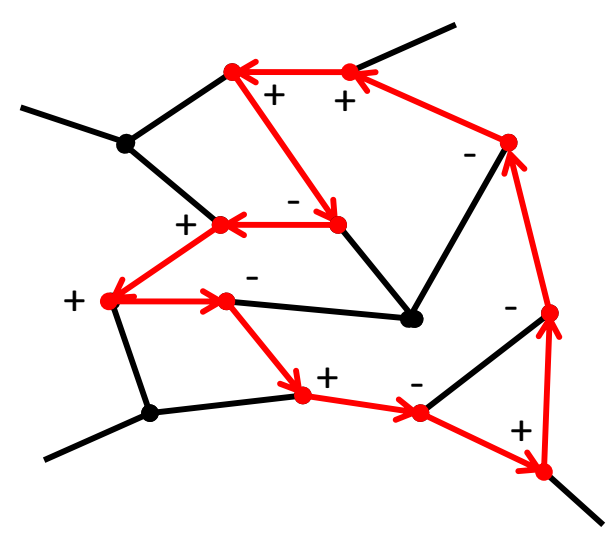

FIG. 6: Illustration of the $\varepsilon_{\boldsymbol{\varsigma}}$ e computation for an imbedded orbit of a homogeneous degree three graph, used in the proof of the Proposition IV.6 Arrow along the marked (red) embedded orbit indicates anti-clockwise (positive) direction of the walk. \pm , standing next to vertices along the embedded orbit (simple cycle), correspond to the values of respective $\varsigma$ contributions. The number of edges adjusted to the \pm vertex and belonging to the interior of the cycle is two/three respectively.

the product of $\varepsilon_{\boldsymbol{\sigma}^{e}}(\partial M)=-1$ (the equality is due to the Kasteleyn nature of $\boldsymbol{\sigma}^{e}$ ) over all faces of $\mathcal{M}$ we arrive at $(-1)^{n_{2}}=(-1)^{n_{1}-l} \varepsilon_{\sigma^{e}}\left(C^{e}\right)$, since each internal edge participates in the product exactly twice and with opposite orientations. Therefore, $\varepsilon_{\boldsymbol{\sigma}^{e}}\left(C^{e}\right)=(-1)^{n_{1}-n_{2}-l}$. The triplet contribution is estimated $\varepsilon_{\mathbf{S}^{e}}\left(C^{e}\right)=(-1)^{n_{R}}$ with $n_{R}$ being the number of right turns of $C^{e}$. Since each internal node of $\mathcal{M}$ is of degree three, whereas a boundary node $a \in C_{0}$ has degree two/three (in $\mathcal{M}$ ) when $C$ turns left/right at $a$, and $\varsigma^{(a) ; e}= \pm 1$, respectively (see Fig. 6 for an illustration). In view of the above argument we have for the number of edges $2 n_{1}=3\left(n_{0}-l\right)+3 n_{R}+2\left(l-n_{R}\right)$. This results in $\varepsilon_{\varsigma^{e}}\left(C^{e}\right)=(-1)^{n_{0}-l}$. Combining the obtained results for the edge and triplet contribution we arrive at $\varepsilon\left(C^{e}\right)=(-1)^{n_{0}-n_{1}+n_{2}}=-1$, with the second equality due to the Euler characteristic relation, $n_{0}-n_{1}+n_{2}=1$.

Corollary IV.7 If an immersed orbit $C$ in $\mathcal{G}$ is lifted to an embedded orbit in $\mathcal{G}^{e}$, then $\varepsilon_{\boldsymbol{\sigma}, \boldsymbol{\varsigma}}(C)=-1$, provided $(\boldsymbol{\sigma}, \boldsymbol{\varsigma})$ is induced.

Proof: Let $C^{e}$ be any lift that satisfies the condition of the corollary. Then $\varepsilon_{\boldsymbol{\sigma}, \boldsymbol{\varsigma}}(C)=\varepsilon_{\boldsymbol{\sigma}^{e}, \boldsymbol{\varsigma}^{e}}\left(C^{e}\right)=-1$ by Propositions IV.5 and IV.6.

\section{B. Induced Orientations Are Suitable}

The following statement explicitly provides a broad class of suitable orientations on an irreducible graph.

Lemma IV.8 Any induced (complete) orientation on an irreducible graph is suitable.

Proof: We are not presenting a complete detailed proof of Lemma IV.8, since it contains a large number of straightforward verification. We rather provide a sequence of steps that leads to a complete proof with brief explanations of these verifications involved on each step. The main idea is to demonstrate that for an induced orientation the binary function $q(\gamma, \boldsymbol{\xi})$, defined in the Definition II.6, does not depend on the partition data $\boldsymbol{\xi}$ (Steps 1 and 2) and then explicitly compute it for some special choices of $\boldsymbol{\xi}$ (Step 3).

Step 1. We start with noting that the triplet component $\boldsymbol{\varsigma}$ of any induced orientation satisfies the following set of local constraints. Let $a$ be an arbitrary vertex of degree 4 or higher, and $\left(b_{0}, b_{1}, b_{2}, b_{3}\right)$ be a set of its four arbitrary distinct neighbors (i.e., $b_{j} \sim a$ ), cyclically ordered in the counterclockwise direction with an arbitrary choice of the first neighbor. Then, the following relation, between the pair-wise components of the left triplet orientations associated with $a$ and $\left(b_{0}, b_{1}, b_{2}, b_{3}\right)$ (see Definition IV.3), holds

$$
\varsigma_{b_{0} b_{2}}^{(a)} \varsigma_{b_{3} b_{1}}^{(a)}=\varsigma_{b_{3} b_{2}}^{(a)} \varsigma_{b_{0} b_{1}}^{(a)} .
$$

This can be verified directly. See Fig. 7 for an illustration.

Step 2. We further introduce a set of local transformations of the decomposition data $\boldsymbol{\xi}$, illustrated in Fig. 7, and show that $q(\gamma ; \boldsymbol{\xi})$ stays invariant under these transformations. Modulo 2 the change in $N(\gamma, \boldsymbol{\xi})$ is totally determined by the change of the intersection index of the edges involved in an elementary transformation. The change in the 


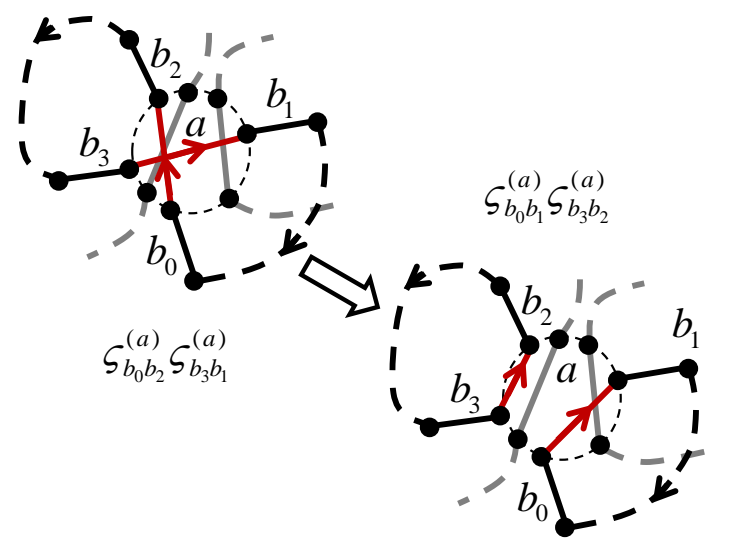

FIG. 7: Example of the elementary transformation which eliminates an intersection of the segments $I_{\left\{b_{0}, b_{2}\right\}}$ and $I_{\left\{b_{1}, b_{3}\right\}}$ shown in red. The products of the triplet orientation factors involved remain the same according to Eq. 12, while the total intersection number of the involved segments with any other (e.g. these shown in gray) remains unchanged modulo 2 . The change in the number of components, $n(\gamma, \boldsymbol{\xi})$, by one is compensated by the identical (modulo 2) change in the intersection index, $N_{a}(\gamma, \boldsymbol{\xi})$.
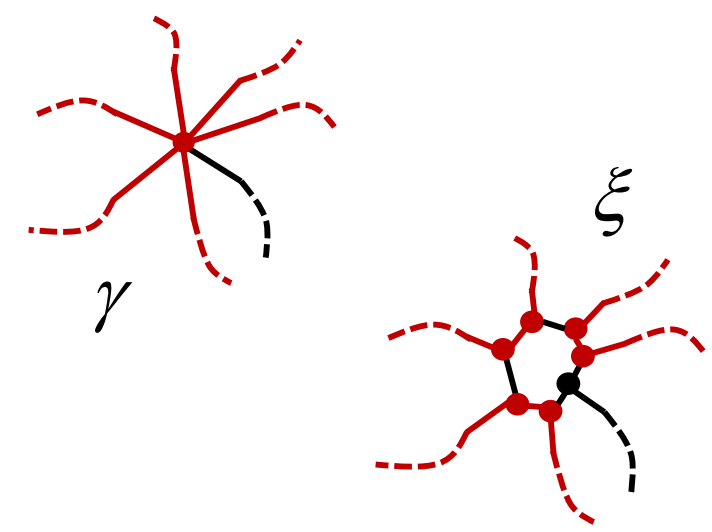

FIG. 8: A single vertex element of an even generalized loop $\left(\mathbb{Z}_{2}\right.$-cycle) $\gamma$ of $\mathcal{G}$ is shown in red at the top/left subfigure. Respected elements of the preferred decomposition data $\xi$ (containing no intersections) lifted to $\mathcal{G}^{e}$ is shown in red at the bottom/right subfigure.

product of the edge and triplet factors involved in the product of all $\varepsilon$-factors in Eq. (8) boils down to the change in the product of two triplet factors involved in the elementary transformation. The change in the number $n(\gamma, \boldsymbol{\xi})$ of the decomposition components can be also analyzed locally, as illustrated in Fig. 7. Therefore, the invariance of $(-1)^{q(\gamma, \boldsymbol{\xi})}$ under an elementary transformation can be verified locally and boils down to the validity of Eq. 12 .

Finally, we note that by performing the described elementary transformation each decomposition data $\boldsymbol{\xi}$ can be transformed to a preferred one, shown in Fig. 8, where for each $a \in \gamma$ the adjacent edges are paired with their nearest neighbors (counterclockwise or clockwise) on $\gamma$. Obviously, any preferred decomposition satisfies the property $N_{a}(\gamma, \boldsymbol{\xi})=0$ for any $a \in \gamma$.

Step 3. The previous step has reduced the proof to the case when the partition data of a decomposed cycle $(\gamma, \boldsymbol{\xi})$ is of a preferred form. We further lift the decomposition components $C_{j}(\gamma, \boldsymbol{\xi})$ to $\mathcal{G}^{e}$ by choosing the connection paths on the extending polygons in a way that they do not intersect with each other as shown in Fig. 8, which is always possible for our special choice of $\boldsymbol{\xi}$. Therefore the immersed orbits $C_{j}(\gamma, \boldsymbol{\xi})$ satisfy the conditions of Corollary IV.7, which results in $q(\gamma, \boldsymbol{\xi})=(-1)^{N(\gamma, \boldsymbol{\xi})}$. Also, as noted above, we have $N_{a}(\gamma, \boldsymbol{\xi})=0$ for all nodes $a \in \gamma$, so that $N(\gamma, \boldsymbol{\xi})=0$.

We would like to emphasize that Lemma IV.8 not only proves the existence of suitable orientations, but rather provides a practical way of building them by reducing the problem to finding Kasteleyn edge orientations on the extended graph. 


\section{GAUGE TRANSFORMATIONS}

Gauge transformation approach discussed in 4, 5, 28, keeping the partition function invariant and the graph intact but modifying the factor functions, also applies to the WBG model. On the other hand, and as shown above, the WBG model is det easy, and thus any binary model derived from WBG models via application of a gauge transformation is also easy. This explains the logic of the main part of this Section - to describe the extended family of binary models reducible via gauge transformations to WBG models. Section VA serves for a brief review of the gauge transformations. Sections $\mathrm{VB} / \mathrm{VC} \mid \mathrm{VD}$ describe the gauge transformations reducing the three noticeable examples of dimer, ice and Ising models to respective WBG models.

Section VE describes a complementary example of an \#X-matching det-tractable model, introduced in [1], which cannot be reduced (to the best of our knowledge) via a standard binary Gauge transformation to a WBG model, but it is still reducible to a WBG model on a contracted graph. The reduction to an easy model is shown here with the help of generalization to intermediate model defined over 3-ary (non-binary) alphabet.

\section{A. Gauge Transformations for general binary model. Reminder.}

Let us first define a general Edge-Binary Graphical (EBG) model on $\mathcal{G}$ with the following partition function

$$
Z_{E B G}=\sum_{\boldsymbol{\pi}} \prod_{a \in \mathcal{G}_{0}} f_{a}\left(\boldsymbol{\pi}_{a}\right),
$$

where $\boldsymbol{\pi}=\left(\pi_{a b}=\pi_{b a}=0,1 \mid\{a, b\} \in \mathcal{G}_{1}\right)$, and $\forall a \in \mathcal{G}_{0}, \boldsymbol{\pi}_{a}=\left(\pi_{a b} \mid b \in \mathcal{G}_{0} ;\{a, b\} \in \mathcal{G}_{1}\right)$ and $f_{a}\left(\boldsymbol{\pi}_{a}\right)$ are the cost functions associated with vertices.

As shown and discussed in [4, 5, 28, the partition function of the general EBG model $(13)$ is invariant under the set of linear, so-called Gauge transformations,

$$
f_{a}\left(\boldsymbol{\pi}_{a}\right) \rightarrow \tilde{f}_{a}\left(\boldsymbol{\pi}_{a}\right)=\sum_{\boldsymbol{\pi}_{a}^{\prime}}\left(\prod_{b \in a} G_{a b}\left(\pi_{a b}, \pi_{a b}^{\prime}\right)\right) f_{a}\left(\boldsymbol{\pi}_{a}^{\prime}\right),
$$

where the newly introduced (two per edge of the graph) Gauge matrices satisfy the following skew orthogonality conditions

$$
\forall\{a, b\} \in \mathcal{G}_{1}: \quad \sum_{\pi} G_{a b}\left(\pi, \pi^{\prime}\right) G_{b a}\left(\pi, \pi^{\prime \prime}\right)=\delta\left(\pi^{\prime}, \pi^{\prime \prime}\right) .
$$

With the gauge transformation applied, the partition function of the model becomes

$$
Z_{E B G}=\sum_{\boldsymbol{\pi}} \prod_{a \in \mathcal{G}_{0}} \tilde{f}_{a}\left(\boldsymbol{\pi}_{a}\right)=\sum_{\boldsymbol{\pi}} \prod_{a \in \mathcal{G}_{0}}\left(\sum_{\boldsymbol{\pi}_{a}^{\prime}}\left(\prod_{b \sim a} G_{a b}\left(\pi_{a b}, \pi_{a b}^{\prime}\right)\right) f_{a}\left(\boldsymbol{\pi}_{a}^{\prime}\right)\right) .
$$

The expression in the middle of Eq. (16) may be considered as a new graphical model. Notice that if all gauge-matrices are non-singular (which is the case discussed in this manuscript) transformation from (13) to (16) is invertible, i.e. for any set of $G$-transformation one naturally defines the reverse $G^{-1}$, bringing us back to the original formulation of Eq. 16.

In [4, 6, 28] the Gauge transformation was discussed in the context of the so-called Belief Propagation (BP)gauge and the resulting Loop Calculus/Series, where BP constraints were imposed additionally to the general (and always maintained) skew-orthogonality constraints (15). The main use of the gauge transformations in the preceding publications consisted in fixing the gauge freedom even further according to the so-called Belief Propagation (BP) conditions. The BP equations, enforcing the respective gauge fixing conditions correspond to fixed points of the Belief Propagation message-passing algorithm popular in physics, computer science and information theory. This special, $\mathrm{BP}$ gauge, reduces the number of terms on the rhs of Eq. 16 requiring that only terms correspondent to $\pi=1$-edges forming a generalized loop (i.e. subgraph with all vertices of degree at least two). The resulting series for partition function consisting of generalized loop only, is called Loop Series.

In the following we show how some set of binary models on the planar graph, which are known to be easy from previous studies, are reduced to WBG forms under respective gauge transformation. 


\section{B. Example: Dimer model as a WBG model}

Gauge transformation allows to restate the original model in a new basis, parameterized by $G$, which is generally a useful freedom to use. To illustrate its general utility, we discuss how applying a gauge transformation allows to restate the dimer model as a WBG model on the same graph. First, let us restate DM in the general EBG form

$$
f_{a}^{(d m)}\left(\boldsymbol{\pi}_{a}\right)=\prod_{b \sim a} w_{a b}^{\pi_{a b} / 2} * \begin{cases}1, & \sum_{c \sim a} \pi_{a c}=1 \\ 0, & \text { otherwise. }\end{cases}
$$

We will build here a gauge transformation around a valid dimer (perfect matching) configuration, $\boldsymbol{p}=\left(p_{a b}=\right.$ $\left.0,1 \mid \forall a: \quad \sum_{b \sim a} p_{a b}=1\right)$. Then introduce the following $\boldsymbol{p}$-dependent gauge $\left(2 \times 2\right.$ matrix with elements $G_{a b}\left(\pi_{a b}, \pi_{a b}^{\prime}\right)$ parametrically dependent on $\boldsymbol{p}$ )

$$
G_{a b}=\left(\begin{array}{ll}
0 & 1 \\
1 & 0
\end{array}\right) \quad \text { if } \quad p_{a b}=p_{b a}=1, \quad \text { or else } \quad G_{a b}=\left(\begin{array}{ll}
1 & 0 \\
0 & 1
\end{array}\right) .
$$

Obviously this gauge satisfies the skew-orthogonality condition 15 . Furthermore, one finds that

$$
\tilde{f}_{a}^{(d m)}\left(\boldsymbol{\pi}_{a} ; \boldsymbol{p}_{a}\right)=\left\{\begin{array}{cc}
\sqrt{w_{a b}}, & \sum_{c \sim a} \pi_{a c}=0 \\
\sqrt{w_{a c}}, & \pi_{a b}=\pi_{a c}=p_{a b}=1, \quad \& \quad \sum_{d \sim a}^{d \neq b, c} \pi_{a d}=0, \\
0, & \text { otherwise, }
\end{array}\right.
$$

where $\{a, b\}$ is the special (unique for vertex $a$ ) matching edge, i.e. $p_{a b}=1$. Therefore DM model (17) is reduced to the WBG model with the following (triplet) weights:

$$
W_{b c}^{(a)}=\left\{\begin{array}{cc}
\omega_{a b}^{p_{a b}-1 / 2} \omega_{a c}^{p_{a c}-1 / 2}, & p_{a b}+p_{a c}=1 \\
0, & \text { otherwise }
\end{array}\right.
$$

Restating it in words, for the new representation an allowed (nonzero) configuration at any vertex corresponds to ones on adjacent edges (there are two of them), of which one is necessarily "active", i.e. present in the perfect matching and thus taking $\pi_{a b}=1$ value. After collecting the $w_{a b}$ pre-factors on the rhs of Eq. 19 into one common multiplier, one observes that this modified model is in fact a particular Wick model (2). The factor functions of the model is identical zero for configurations with at least two adjacent edges taking "active" $\pi=1$ value. Notice also that the WBG representation for the dimer model is not unique as it can be build around any valid dimer configurations. Evidently any two valid representations, described by the gauges $G_{1}$ and $G_{2}$ can be transformed to each other via respective gauge transformations, $G_{2} G_{1}^{-1}$.

We conclude this Subsection mentioning briefly yet another alternative reduction of the dimer model to a WBG model, utilizing a topological transformation (specifically a contraction) of the original graph $\mathcal{G}$. Consider a valid dimer configuration $\boldsymbol{p}_{0}$ as a set of edges $\{a, b\} \in \mathcal{G}_{1}$ and contract each edge $\{a, b\} \in \boldsymbol{p}_{0}$ to a point. This results in a new graph $\overline{\mathcal{G}}$, whose nodes $\overline{\mathcal{G}}_{0}=\boldsymbol{p}_{0}$ are represented by the edges that belong to the reference dimer configurations, and whose edges $\overline{\mathcal{G}}_{1}=\mathcal{G}_{1} \backslash \boldsymbol{p}_{0}$ are represented by the rest of the edges of $\mathcal{G}$. Each valid dimer configuration $\boldsymbol{p}$ on $\mathcal{G}$ generates a configuration $\boldsymbol{\pi}$ of a binary edge model on $\overline{\mathcal{G}}$ that has a form $\pi_{\alpha}=1$ if $\alpha \in \boldsymbol{p}$, and $\pi_{\alpha}=0$, otherwise. (Here we consider an edge $\alpha \in \overline{\mathcal{G}}_{1}=\mathcal{G}_{1} \backslash \boldsymbol{p}_{0} \subset \mathcal{G}_{1}$ of $\overline{\mathcal{G}}$ as an edge of $\mathcal{G}$.) It is easy to see that a vertex function $f_{a}\left(\boldsymbol{\pi}_{a}\right)$ for $a \in \overline{\mathcal{G}}_{0}$ is nonzero only when all components of $\boldsymbol{\pi}_{a}$ are zeros (the case $a \in \boldsymbol{p}_{0}$ ), or when exactly two components have the value one (otherwise), and also that the local weights satisfy the conditions of the Wick binary model expressed in Eq. (3).

\section{Example: Ice Model for graphs of degree three}

The ice model is defined in terms of orientations of edges on the graph. A valid configuration/orientation is such that no vertex has adjusted edges all oriented onwards or inwards. Here we consider the case when all vertices on $\mathcal{G}$ are of degree three. (Without loss of generality our consideration here can be extended to vertices of degree not larger than three.) Weight of any allowed orientation of the graph is unity. This model, \#PL-3-NAE-ICE according to the complexity theory classification, was discussed in [1, 3] in lieu of its reduction to dimer model via a holographic algorithm. Related models were studied extensively in the mathematical physics literature $22,24$. 
The ice model can be conveniently restated in terms of normal binary variables ${ }^{5}$. This will require a graphical transformation consisting in breaking every edge, $\{a, b\}$, by inserting a new auxiliary vertex $a-b$. Then, binary variables assigned to the pair of new edges are $\pi_{a, a-b}=0, \pi_{b, a-b}=1$ if the direction of the arrow (for the original orientation of the edge) was $a \rightarrow b$ and $\pi_{a, a-b}=1, \pi_{b, a-b}=0$ for the $b \rightarrow a$ arrow/orientation. The partition function of the respective binary model becomes

$$
\begin{aligned}
& Z_{i c e}=\sum_{\boldsymbol{\pi}^{\prime}}\left(\prod_{a \in \mathcal{G}_{0}} f_{a}\left(\boldsymbol{\pi}_{a}\right)\right)\left(\prod_{\{a, b\} \in \mathcal{G}_{1}} g_{a-b}\left(\pi_{a, a-b}, \pi_{b, a-b}\right)\right), \\
& f_{a}\left(\boldsymbol{\pi}_{a}^{\prime}\right)=\left\{\begin{array}{ll}
1, \exists b, c \sim a, & \text { s.t. } \pi_{a, a-b} \neq \pi_{a, a-c} \\
0, & \text { otherwise }
\end{array},\right. \\
& g_{a-b}\left(\boldsymbol{\pi}_{a}^{\prime}\right)=\left\{\begin{array}{cc}
1 & \pi_{a, a-b} \neq \pi_{b, a-b} \\
0, & \text { otherwise }
\end{array},\right.
\end{aligned}
$$

where $\boldsymbol{\pi}^{\prime}=\boldsymbol{\pi} \cup\left(\pi_{a, a-b}=0,1 \mid\{a, b\} \in \mathcal{G}_{1}\right)$ and $\boldsymbol{\pi}_{a}^{\prime}=\left(\pi_{a, a-b}=0,1 \mid b \in \delta_{\mathcal{G}}(a)\right)$.

Let us introduce the following gauge transformation on all edges of the newly formed extended graph

$$
G_{a, a-b}^{(i c e)}=\frac{1}{\sqrt{2}}\left(\begin{array}{cc}
1 & 1 \\
-1 & 1
\end{array}\right)
$$

Factor functions of the ice model in the new basis becomes

$$
\begin{aligned}
& \tilde{f}_{a}\left(\pi_{a, a-1}, \pi_{a, a-2}, \pi_{a, a-3}\right)=\frac{3}{\sqrt{2}} *\left\{\begin{array}{cc}
1, & \pi_{a, a-1}=\pi_{a, a-2}=\pi_{a, a-3}=0 \\
-1 / 3, & \sum_{i} \pi_{a, a-i}=2 \\
0, & \text { otherwise }
\end{array},\right. \\
& \tilde{g}_{a-b}\left(\boldsymbol{\pi}_{a}^{\prime}\right)=\left\{\begin{array}{cc}
1, & \pi_{a, a-b}=\pi_{b, a-b}=0 \\
-1, & \pi_{a, a-b}=\pi_{b, a-b}=1 \\
0, & \text { otherwise }
\end{array}\right.
\end{aligned}
$$

where $(a, a-i)$ with $i=1,2,3$ marks three edges adjusted to vertex $a$. Accumulating the product of the overall $3 / \sqrt{2}$ terms into a common (normalization) multiplier, one immediately finds that the resulting model is of the Wick's type (2) where

$$
\forall \alpha \neq \beta, \quad \alpha, \beta=a-1, a-2, a-3: \quad W_{\alpha, \beta}^{(a)}=-1 / 3 ; \quad \forall a \sim b: \quad W_{a b}^{(a-b)}=-1 .
$$

Indeed, given that $\forall a \in \mathcal{G}\left|\delta_{a}(\mathcal{G})\right|<4$, one simply does not have any $W^{(a)}$ terms in the ice model version of 2 (2) with $\left|\delta_{a}(\mathcal{G})\right|>4$, and the only nontrivial vertices are these with $\left|\delta_{a}(\mathcal{G})\right|=2$. Notice, that this Wick feature would not apply to the ice model on planar graphs with vertices of degree higher than three - one can still use the gauge transformation but the resulting transformed model will not maintain Wick's property.

Note that transformation of the ice model, identical to the one discussed above (e.g. utilizing some alternative terminology) was also used as a show case of the holographic algorithms of Valiant in [1-3].

\section{Example: Ising Model}

We consider the classical Ising model without magnetic field:

$$
Z_{I}=\sum_{\boldsymbol{\sigma}} \exp \left(\sum_{\{a, b\} \in \mathcal{G}_{0}} \sigma_{a} J_{a b} \sigma_{b}\right),
$$

\footnotetext{
${ }^{5}$ An alternative derivation, not requiring a graphical transformation, is discussed in Appendix B
} 


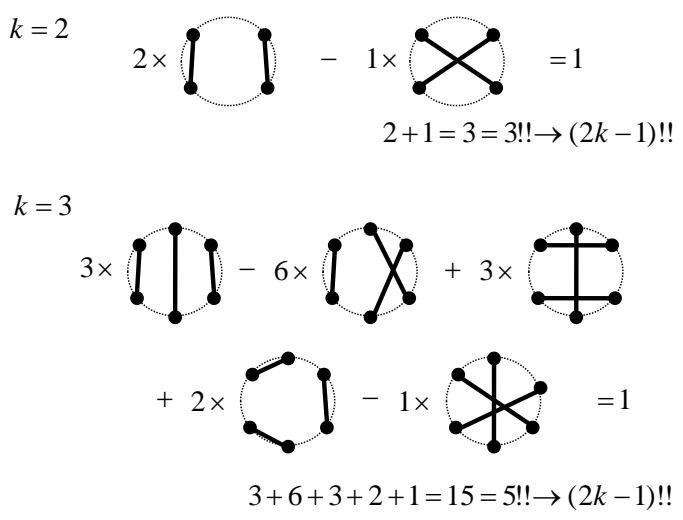

FIG. 9: Illustration clarifying the Wick-feature of Eq. 32.

where $\boldsymbol{\sigma}=\left(\sigma_{a}= \pm 1 \mid a \in \mathcal{G}_{0}\right)$. The original formulation $(28)$ is in terms of binary variables associated with vertices, however the following EBG representation is more appropriate for our purposes

$$
\begin{aligned}
& Z_{I}=\sum_{\pi^{\prime}}\left(\prod_{a \in \mathcal{G}_{0}} f_{a}(\boldsymbol{\pi})\right)\left(\prod_{\{a, b\} \in \mathcal{G}_{1}} g_{a b}\left(\pi_{a, a-b}, \pi_{b, a-b}\right)\right), \\
& f_{a}(\boldsymbol{\pi})=\left\{\begin{array}{l}
1, \forall b, c \in \delta_{\mathcal{G}}(a) \pi_{a, a-b}=\pi_{a, a-c}, \\
0, \quad \text { otherwise }
\end{array},\right. \\
& g_{a b}\left(\pi_{a, a-b}, \pi_{b, a-b}\right)=\left\{\begin{array}{cc}
\gamma_{a b}, & \pi_{a, a-b}=\pi_{b, a-b} \\
\mu_{a b}, & \pi_{a, a-b} \neq \pi_{b, a-b}
\end{array},\right.
\end{aligned}
$$

where $\gamma_{a b}=\exp \left(J_{a b}\right), \mu_{a b}=\exp \left(-J_{a b}\right)$ and we adopted notations of the previous Subsection for new (auxiliary) vertices breaking each edge of the original edge in two.

Applying the Gauge transformation (24), introduced above for the Ice model, to the Ising model one arrives at the following factor functions in the new basis

$$
\begin{aligned}
& \tilde{f}_{a}(\boldsymbol{\pi})=2^{-\left|\delta_{\mathcal{G}}(a)\right| / 2+1} *\left\{\begin{array}{cc}
1, & \sum_{b \sim a} \pi_{a, b-a}=0 \bmod 2, \\
0, & \text { otherwise }
\end{array}\right. \\
& \tilde{g}_{a b}\left(\pi_{a, a-b}, \pi_{b, a-b}\right)=\left(\gamma_{a b}+\mu_{a b}\right) *\left\{\begin{array}{cc}
1, & \pi_{a, a-b}=\pi_{b, a-b}=0 \\
\left(\gamma_{a b}-\mu_{a b}\right) /\left(\gamma_{a b}+\mu_{a b}\right), & \pi_{a, a-b}=\pi_{b, a-b}=1 \\
0, & \text { otherwise }
\end{array}\right.
\end{aligned}
$$

With the product of all the pre-factor terms, $2^{-\left|\delta_{\mathcal{G}}(a)\right| / 2+1}$ and $\left(\gamma_{a b}+\mu_{a b}\right)$, accounted for in the overall multiplier, the resulting model is one of the WBG models of (2). This Wick feature of the modified graphical model is obvious for degree two vertices of Eq. (33), and the only part which requires additional clarification concerns the relation between a configuration of Eq. 32 with $\sum_{b \sim a} \pi_{a, b-a}>2$ and its pairwise decomposition. Indeed, for a $\mathbb{Z}_{2}$-cycle $\gamma$ and a vertex $a \in \gamma_{0}$ of valence $\left|\delta_{\gamma}(a)\right|=2 k$ with respect to $\gamma$, there are $(2 k-1)$ !! local partitions with $((2 k-1) ! !+1) / 2$ and $((2 k-1) ! !-1) / 2$ of them having modulo two intersection index equal to zero and one, respectively. Therefore according to the Wick rules, the cumulative local contribution associated with the vertex $a$ is, $((2 k-1) ! !+1) / 2-$ $((2 k-1) ! !-1) / 2=1$, that is fully consistent with Eq. $(32)$. This simple combinatorial relation is illustrated in Fig. 9 for $k=2$ and $k=3$. Summarizing, the Ising model is reduced to the WBG model with the following characteristics (W-weights):

$$
\forall b \neq c, b, c \sim a: \quad W_{b-a, c-a}^{(a)}=0 ; \quad \forall a \neq b: \quad W_{a, b}^{(a-b)}=\frac{\gamma_{a b}-\mu_{a b}}{\gamma_{a b}+\mu_{a b}} .
$$

\section{E. \#X-matching model}

The \#X-matching model is defined as follows [1-3]: On a planar bipartite graph, where $V_{1}$ and $V_{2}$ are bi-partitions of the full set of nodes and the nodes in $V_{1}$ all have degree 2, consider matchings (monomer-dimer configurations) 
weighted in a way that all dimers (edges) have arbitrary weights, monomers on $V_{1}$ are all of unit weight and the weights of the monomers on $V_{2}$ are equal to minus the sum of the dimer weights of edges emanated from the vertex.

It is convenient to view the bipartite graph in the original definition of the \#X-matching model as a graph with the sets of nodes $V$ and edges $E$ represented by $V=V_{2}$ and $E=V_{1}$, respectively. We will adopt notations similar to these used in Section VC, devoted to the Ice model, calling $a, b, \cdots$ the vertices of $V=V_{2}$ and $a-b, \cdots$ vertices belonging to $E=V_{1}$. Then the partition function $Z_{\# X}$ of the $\# X$-matching model has the following EBG representation on the graph $\mathcal{G}=\left(\mathcal{G}_{0}, \mathcal{G}_{1}\right)=(V, E)$

$$
\begin{aligned}
& Z_{\# X}=\sum_{\boldsymbol{\pi}}\left(\prod_{a \in \mathcal{G}_{0}} f_{a}\left(\boldsymbol{\pi}_{a}\right)\right)\left(\prod_{\{a, b\} \in \mathcal{G}_{1}} g_{a b}\left(\pi_{a, a-b}, \pi_{b, a-b}\right)\right), \\
& f_{a}\left(\boldsymbol{\pi}_{a}\right)=\left\{\begin{array}{cc}
-\sum_{b \sim a} w_{a b}, & \forall b \sim a \\
\sum_{b \sim a} w_{a b} \pi_{a, a-b}, & \sum_{b \sim a} \pi_{a, a-b}=0, \\
0, & \text { otherwise }
\end{array},\right. \\
& g_{a b}\left(\pi_{a, a-b}, \pi_{b, a-b}\right)=\left\{\begin{array}{c}
0, \quad \pi_{a, a-b}=\pi_{b, a-b}=1 \\
1, \pi_{a, a-b}=0 \text { or } \pi_{b, a-b}=0
\end{array}\right.
\end{aligned}
$$

Our task in this Subsection is to show via a gauge-transformation approach, alternative to the match-gate approach of [1 3], that the partition function of the \#X-matching model, defined as the weighted number of the monomerdimer configurations, is det-easy. To achieve this goal we will need to introduce, as in the above Subsection, a gauge transformation reducing the model to another model known to be det-easy. However, the gauge transformation of this Subsection is principally different from these discussed in the rest of the paper, as here we will need to consider generalization from binary-setting to a $q$-ary, with $q=3$, alphabet. For this purpose we restate Eqs. 35 37) as the following 3-ary Edge Graphical Model over the set of variables $\tilde{\boldsymbol{\pi}}=\left\{\tilde{\pi}_{a, a-b}\right\}$ that attain the values $\tilde{\pi}_{a, a-b}=0, \pm 1$ :

$$
\begin{aligned}
& Z_{\# X}=\sum_{\tilde{\boldsymbol{\pi}}}\left(\prod_{a \in \mathcal{G}_{0}} \bar{f}_{a}\left(\tilde{\boldsymbol{\pi}}_{a}\right)\right)\left(\prod_{\{a, b\} \in \mathcal{G}_{1}} \bar{g}_{a b}\left(\tilde{\pi}_{a, a-b}, \tilde{\pi}_{b, a-b}\right)\right), \\
& \bar{f}_{a}\left(\tilde{\boldsymbol{\pi}}_{a}\right)=\left\{\begin{array}{cc}
1, & \forall b \sim a \quad \tilde{\pi}_{a, a-b}=0 \\
w_{a b} \tilde{\pi}_{a, a-b}, & \tilde{\pi}_{a, a-b}= \pm 1 \quad \text { and } \forall c \neq b \quad \tilde{\pi}_{a, a-c}=0 \\
0, & \text { otherwise }
\end{array}\right. \\
& \bar{g}_{a b}\left(\tilde{\pi}_{a, a-b}, \tilde{\pi}_{b, a-b}\right)=\left\{\begin{array}{cc}
0, & \tilde{\pi}_{a, a-b}=\tilde{\pi}_{b, a-b}=1 \\
1, & \text { otherwise }
\end{array} .\right.
\end{aligned}
$$

To see equivalence between the binary and $q$-ary representations let us walk backwards, from Eqs. 35. 37) to Eqs. 38. 40, and associate with any configuration $\tilde{\boldsymbol{\pi}}$ of our 3 -ary model a binary configuration $\boldsymbol{\pi}$ by setting $\pi_{a, a-b}=1$ when $\tilde{\pi}_{a, a-b}=1$, and $\pi_{a, a-b}=0$ when $\tilde{\pi}_{a, a-b}=0,-1$. Then, we do partial summation over all $\tilde{\pi}_{a, a-b}=0,-1$ correspondent to $\pi_{a, a-b}=0$. In a general case the result of such partial summation would be non-local with respect to the graph. However, in our special case the resulting model happens to be graph-local. Indeed, the weights $\bar{g}_{a b}\left(\tilde{\pi}_{a, a-b}, \tilde{\pi}_{b, a-b}\right)$ in Eq. 40 actually depend on the reduced variables $\pi_{a, a-b}$ and $\pi_{b, a-b}$ and the partial summation can be performed for each node $a \in \mathcal{G}_{0}$ independently. Finally, we arrive at the binary formulation of Eqs. (35.37).

One may wonder why changing from binary to 3-ary representation is useful? In fact it is very useful because of a greater freedom of further transformation the 3-ary representation offers. Indeed, we will see below that relating $\tilde{\pi}$ variables to $\pi$ variables in a new way allows to reduce the 3-ary model to the already known det-easy model (specifically, a dimer model, already shown reducible to a WBG model in Section VB).

Namely, we set $\pi_{a, a-b}=1$ when $\tilde{\pi}_{a, a-b}= \pm 1$, and $\pi_{a, a-b}=0$ when $\tilde{\pi}_{a, a-b}=0$. The resulting binary model turns out to be the dimer model on the reduced version of $\mathcal{G}$ (no half-edges only full edges) with the dimer weights $-w_{a b} w_{b a}$ for the $\{a, b\}$ edges. This follows from the following arguments. Consider a link $\{a, b\}$. If $\pi_{a, a-b}=1$ and $\pi_{b, a-b}=0$ we have $\tilde{\pi}_{a, a-c}=0$ for all $c \sim a$ with $c \neq b$, and also $\bar{g}_{a b}\left(\tilde{\pi}_{a, a-b}, \tilde{\pi}_{b, a-b}\right)=1$, so that the partial summation over $\tilde{\pi}_{a, a-b}= \pm 1$ can be performed independently, which results in 0 . If $\pi_{a, a-b}=\pi_{b, a-b}=1$, we have $\tilde{\pi}_{a, a-c}=0$ for all $c \sim a$ with $c \neq b$, and also $\tilde{\pi}_{b, b-c}=0$ for all $c \sim b$ with $c \neq a$. This allows the summations over $\pi_{a, a-b}= \pm 1$ and $\pi_{b, a-b}= \pm 1$ to be performed independently, thus resulting in the factors of 0 for $\pi_{a, a-b}=\pi_{b, a-b}=1$, and $w_{a b} w_{b a} \pi_{a, a-b} \pi_{b, a-b}$, otherwise. QED.

It is worth to note that in terms of the language of loop towers for $q$-ary models, developed in our earlier work on loop calculus for $q$-ary alphabets [28, the partial re-summation based on partitioning the alphabet into one special letter $(\tilde{\pi}=0)$ and the rest $(\tilde{\pi}= \pm 1)$ should be viewed as a partial gauge fixing that corresponds to the lowest level of the loop tower. 


\section{DISCUSSION AND FUTURE PLANS}

In the present manuscript we have identified a broad class of models that allow for an easy solution on planar graphs. Our approach is based on the gauge invariance of vertex models on graphs that has been implemented in our previous work in the context of Belief Propagation and Loop Series and the notion of a Wick vertex model, referred to as WBG. The WBG models are described in terms of an arbitrary choice of weights associated with edges of the graph, and explicit construction of the high-degree (even coloring) vertex weights from the respective edge weights according to the so-called Wick rules. We have established an equivalence between the WBG models and the Gaussian fermion (Grassmann variables based) models on the same graph, referred to as the $\mathrm{VG}^{3}$ models (Theorem II.7). The models are "easy" as their partition functions are equal to Pfaffians of square matrices of the size equal to twice the number of edges of the graph. We have also demonstrated that the dimer model that constitutes the "solving tool" of the holographic algorithm of Valiant [1 3 ] is gauge equivalent to a WBG model. The other two components of the holographic approach include graphical transformations (extensions of the original graph) and linear transformations, which can be described as particular cases of gauge transformations using the language adopted in this manuscript. If one represents the partition function of the dimer model on the extended graph, obtained by using the gadgets of the holographic algorithm, as a Gaussian Grassmann integral and integrate over the variables related to the new vertices that result from the graph extension, the result will be represented by a Gaussian Grassman integral on the original graph. Therefore, our approach provides an explicit and invariant description of the models that can be treated using the holographic algorithm as the models gauge equivalent to WBG models. It also provides with an alternative way to obtain an easy solution, while sticking to the original graph all the time.

Some problems we plan to address in the future, extending on the results/approach described in the manuscript, are:

- Generalize the approach to account for det-easy planar graphical models defined over $q$-ary alphabet with $q>2$. As illustrated in Subsection VE there exist an interesting new class of more general $q$-ary models which are reducible to det-easy binary graphical models.

- Use the described hierarchy of easy planar models as a basis for efficient variational approximation of planar but difficult problems, and possibly more generally non-planar models. Then, on the next level, build a controlled perturbative corrections to the variational result. Notice, that this approach may also be useful for building efficient variational matrix-product state wave functions for quantum planar models, e.g. in the spirit of [29].

- Analysis of Wick Gaussian models on surface graphs of nonzero genus. This work will extend the classical results [15, 30 35] stating that partition function of dimer models on graphs embedded in surface of genus $g$, so-called surface graphs, is expressed as a sum over $2^{2 g}$ Pfaffians, each correspondent to a "spinor" structure parameterizing the equivalence classes of Kasteleyn orientations on the locally planar graphs. The approach developed in this manuscript allows for a relatively straightforward extension to the case of surface graphs. In particular, we plan to show in a forthcoming publication that Kasteleyn orientations on the extended graph $\mathcal{G}^{e}$ generate all $2^{2 g}$ spinor structures on the embedding Riemann surface.

- Study Wick Gaussian models on non-planar but Pfaffian orientable or $k$-Pfaffian orientable graphs (thus any dimer model on surface graph of genus $g$ is $2^{2 g}$-Pfaffian orientable), also utilizing the new results from graph theory on the Pfaffian orientability [36].

- For the case of a general EBG model we will apply the statistical variational principle to build the "best" approximation in a form of a WBG model and address the problem of finding efficient ways to account for the corrections.

\section{ACKNOWLEDGMENTS}

We are thankful to David Gamarnik for attracting our attention to the holographic algorithms of [1-3], to John R. Klein, Jason Johnson and Vicenc Gomez for useful discussions and comments, and also to an anonymous Referee whose comments force us to reconsider (and hopefully improve) the presentation sequence. This material is based upon work supported by the National Science Foundation under CHE-0808910 (VC) and CCF-0829945 (MC via NMC). The work at LANL was carried out under the auspices of the National Nuclear Security Administration of the U.S. Department of Energy at Los Alamos National Laboratory under Contract No. DE-AC52-06NA25396. MC 
also acknowledges partial support of KITP at UCSB where some part of this work was done.

[1] L. G. Valiant, SIAM Journal on Computing 31, 1229 (2002), URL http://link.aip.org/link/?SMJ/31/1229/1.

[2] L. Valiant, Foundations of Computer Science, 2004. Proceedings. 45th Annual IEEE Symposium on pp. 306-315 (2004).

[3] L. G. Valiant, SIAM Journal on Computing 37, 1565 (2008).

[4] M. Chertkov and V. Chernyak, Physical Review E (Statistical, Nonlinear, and Soft Matter Physics) 73, 065102 (pages 4) (2006), URL http://link.aps.org/abstract/PRE/v73/e065102.

[5] M. Chertkov and V. Y. Chernyak, Journal of Statistical Mechanics: Theory and Experiment 2006, P06009 (2006), URL http://stacks.iop.org/1742-5468/2006/P06009

[6] M. Chertkov, V. Y. Chernyak, and R. Teodorescu, Journal of Statistical Mechanics: Theory and Experiment 2008, P05003 (19pp) (2008), URL http://stacks.iop.org/1742-5468/2008/P05003

[7] V. Y. Chernyak and M. Chertkov, J. of Stat. Mech. p. P12011 (2008), URL http://arxiv.org/abs/0809.3479

[8] V. Y. Chernyak and M. Chertkov, J. of Stat. Mech. p. P12012 (2008), URL http://arxiv.org/abs/0809.3481

[9] L. Onsager, Phys. Rev. 65, 117 (1944).

[10] M. Kac and J. Ward, Physical Review 88 (1952).

[11] N. V. Vdovichenko, Soviet Phys. JETP 20, 477 (1965).

[12] H. N. V. Temperley and M. Fisher, Philosophical Magazine 6, 1061 (1961).

[13] M. Fisher, Journal of Mathematical Physics 710 (1966).

[14] P. W. Kasteleyn, Physica 27, 1209 (1961).

[15] P. W. Kasteleyn, Journal of Mathematical Physics 4, 287 (1963), URL http://link.aip.org/link/?JMP/4/287/1

[16] P. Kasteleyn, in Graph theory and theoretical physics (Academic Press, New York, 1967).

[17] S. A. Cook, in STOC 'r1: Proceedings of the third annual ACM symposium on Theory of computing (ACM, New York, NY, USA, 1971), pp. 151-158.

[18] R. M. Karp, in Complexity of Computer Computations, edited by R. E. Miller and J. W. Thatcher (Plenum Press, 1972), pp. $85-103$.

[19] F. Barahona, J. Phys. A 15 (1982).

[20] L. G. Valiant, Theoret. Comput. Sci. 8, 189 (1979).

[21] S. P. Vadhan, SIAM Journal on Computing 31, 398 (1997).

[22] E. H. Lieb, Phys. Rev. Lett. 18, 692 (1967).

[23] E. H. Lieb, Phys. Rev. 162, 162 (1967).

[24] R. J. Baxter, Exactly Solved Models in Statistical Mechanics (Dover Publications, 2007), ISBN 0486462714.

[25] V. Popov, Functional integrals in quantum field theory and statistical physics (Kluwer Academic Publ, 1983).

[26] L.Lovász and M. Plummer, Matching Theory (Academic Press, 1986).

[27] T. Morita, Physica A Statistical Mechanics and its Applications 144, 118 (1987).

[28] V. Y. Chernyak and M. Chertkov, Information Theory, 2007. ISIT 2007. IEEE International Symposium on pp. 316-320 (2007), URL http://arxiv.org/abs/cs.IT/0701086

[29] F. Verstraete and J. I. Cirac, Physical Review B (Condensed Matter and Materials Physics) 73, 094423 (pages 8) (2006), URL http://link.aps .org/abstract/PRB/v73/e094423

[30] T. Regge and R. Zecchina, Journal of Mathematical Physics 37, 2796 (1996).

[31] A. Galluccio and M. Loebl, Electronic Journal of Combinatorics, 6(1). Research Paper 6 p. 18 (1999).

[32] A. Galluccio and M. Loebl, Electronic Journal of Combinatorics, 6(1). Research Paper 7 p. 7 (1999).

[33] T. Regge and R. Zecchina, Journal of Physics A Mathematical General 33, 741 (2000).

[34] D. Cimasoni and N. Reshetikhin, Communications in Mathematical Physics 275, 187 (2007), URL http://www. springerlink.com/content/a2m2385607g31614.

[35] D. Cimasoni and N. Reshetikhin, Communications in Mathematical Physics 281, 445 (2008), URL http://www. springerlink.com/content/74454243n7281kv6.

[36] R. Thomas, in Proceedings of the International Congress of Mathematics (2006), URL http://www.math.gatech.edu/ $\sim$ thomas/PAP/pfafsurv.pdf

[37] A. Haefliger, in Proceedings of Liverpool Singularities Symposium, II (Lecture Notes in Math., Vol. 209, Springer, Berlin, 1970), pp. $128-141$.

[38] M. A. Bershadsky and A. A. Migdal, Physics Letters B 174, 393 (1986), ISSN 0370-2693, URL http://www. sciencedirect.com/science/article/B6TVN-46YSTRG-26P/2/54ee019032c3113e3fbba0b8a9b145ee

[39] L. Bogacz, Z. Burda, J. Jurkiewicz, A. Krzywicki, C. Petersen, and B. Petersson, Acta Physica Polonica B 32, 4121 (2001), arXiv:hep-lat/0110063.

\section{Appendix A: Topology behind the door: Self-intersection invariant of immersions and spinor structures}

The main result of this manuscript is represented by Theorem II.7. The proof of the Theorem rests on a computation of the relevant Gaussian Grassmann integral using a certain expansion, combined with the Wick Theorem 


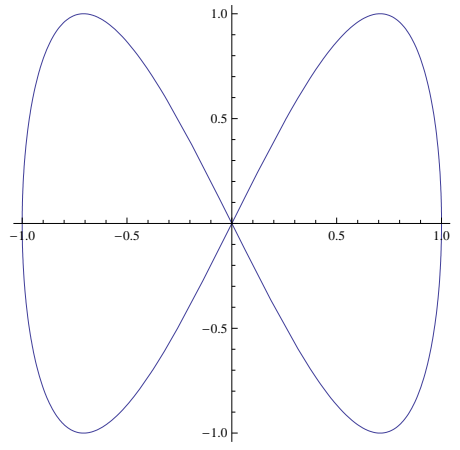

(a)An example of a smooth closed trajectory. Parametrization chosen for plotting is $\boldsymbol{r}=$ $\left(r_{1}(t), r_{2}(t)\right)=(\sin (t), \sin (2 t))$, where $t$ changes from 0 to $2 \pi$.

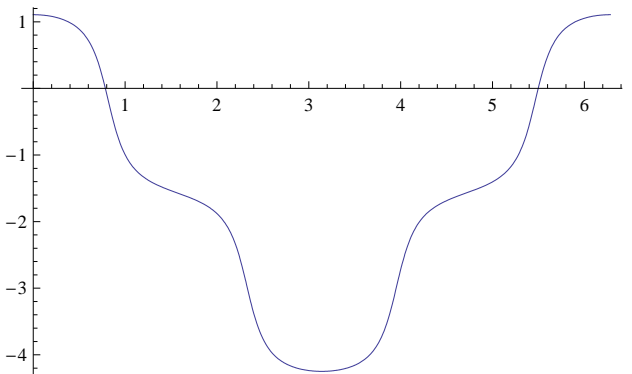

(b)Direction of velocity (angle), $\varphi(t)=\arctan (2 \cos (2 t) / \cos (t))$, for a particle moving along the immersed orbit from (A), thus $\boldsymbol{r}$ are coordinates of the particle in $\mathbb{R}^{2}$, as a function of "time" $t$. The invariant, measuring total number of angular rotations, is 0 for this example.

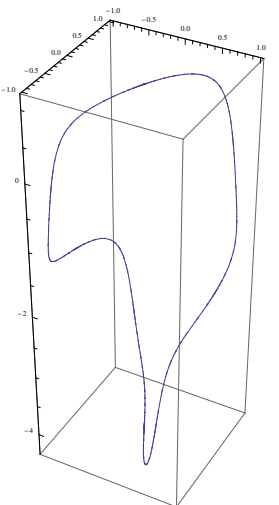

(c) Trajectory of the particle in the phase-space, $\boldsymbol{x}=$ $\left(r_{1}(t), r_{2}(t), \varphi(t)\right) \in$ $M^{3}=\mathbb{R}^{2} \times S^{1}$.

FIG. 10: Illustration of an immersion in $\mathbb{R}^{2}$ of an orbit with one crossing and its phase-space (particle) counterpart.

and Lemma IV.8 In this Appendix we present a brief qualitative discussion (without proofs) of the topology that stands behind the presented combinatorial proofs presented in the main body of the manuscript. This will connect our results to the approach developed in [34, 35] for the dimer model on surface graphs, and also provide some ideas on how our results on the equivalence between the Wick and binary models can be extended to the surface graph case (the details of this description and related proofs will be published elsewhere).

A topological nature of the equivalence between the WBG and $G^{3}$ models can be seen by comparing Eqs. (9) and (10) that play a major role in the proof of Theorem II.7. Each contribution in both sums can be represented as a product of the individual contributions labeled by the immersed orbits $C_{j}(\gamma, \boldsymbol{\xi})$. The individual contribution associated with an immersed orbit $C$ has the same absolute value for both expansions, they differ by the sign factors only. For the $\mathrm{G}^{3}$ model the sign associated with an immersed orbit $C$ is $-\varepsilon(C)=(-1)^{s(C)+1}$ (where the last expression defines $s(C)$ as a function of $\varepsilon(C)$ introduced in the main text), in the WBG model case the sign is $(-1)^{N(C)}$ with $N(C)$ being the number of self-intersections of $C$. Note that the intersections between different orbits in the expansion of $\gamma$, although apparently affecting the sign factor in Eq. (9), can be actually eliminated from the consideration, since in the planar case two orbits always intersect at an even number of points. In this appendix we will argue that $s(C)$ can be associated with the unique spinor structure on $\mathbb{R}^{2}$, and the equivalence between the models originate from the topological formula, $N(C)+1=s(C)(\bmod 2)$, that relates the self-intersection number to the spinor structure.

We proceed with more precise formulations. Note that the statement of Lemma IV.8 can be interpreted in the following way: The function $q(\gamma, \boldsymbol{\xi})$ of $\gamma$ and $\boldsymbol{\xi}$ defined by Eq. (8) can be viewed as a $\mathbb{Z}_{2}$-valued function defined on the sets of immersed orbits, represented by $C_{j}(\gamma, \boldsymbol{\xi})$, and it satisfies the property $q(\gamma, \boldsymbol{\xi})=0 . q(\gamma, \boldsymbol{\xi})$ consists of three contributions: the number $N(\gamma, \boldsymbol{\xi})$ of intersections and self-intersections of the involved orbits (naturally modulo two), the number $n(\gamma, \boldsymbol{\xi})$ of the orbits involved, and the contributions $s\left(C_{j}(\gamma, \boldsymbol{\xi})\right)$ that arise from the individual orbits, where $s \equiv(\log (\varepsilon) /(i \pi)$. What we intend to illustrate is that by combining the modulo two numbers of intersections and the modulo two number of orbits in a decomposition of a planar $\mathbb{Z}_{2}$-cycle $\gamma$ we arrive at a topological invariant $p(\gamma, \xi)=N(\gamma, \boldsymbol{\xi})+n(\gamma, \boldsymbol{\xi}) \in \mathbb{Z}_{2}$, referred to as the self-intersection invariant, whereas the individual orbit contributions $s\left(C_{j}(\gamma, \boldsymbol{\xi})\right)$ and the corresponding sign factors $\varepsilon=(-1)^{s}$ are associated with a spinor structure on the plane $\mathbb{R}^{2}$, the original planar graph and its extension are imbedded into. Combining the self-intersection invariant with the spinor structure related contributions we arrive at the $\mathbb{Z}_{2}$-invariant $q=p+s$ that depend on the planar $\mathbb{Z}_{2}$-cycle $\gamma$ only, and $q=0$, since all cycles in $\mathbb{R}^{2}$ are contractable. This will be done by interpreting the orbits as closed trajectories of a free particle in the plane, also referred to as a free planar particle.

\section{Immersions, self-intersection invariant and spinor structures on $\mathbb{R}^{2}$}

This Subsection introduces some useful objects that are new to the manuscript, as defined for continuous spaces, e.g. $\mathbb{R}^{2}$, rather than for the graphical structure as in the main part of the manuscript. This continuous spaces approach 
will be used in the following Subsection for a topological illustration of Lemma IV.8. The arguments presented there can be easily converted into a proof, however, this task goes beyond the scope of this manuscript. We will start with introducing a special type of smooth maps (trajectories) $C: S^{1} \rightarrow \mathbb{R}^{2}$, referred to as immersions and denoted $C: S^{1} \rightarrow \mathbb{R}^{2}$. The main reason for bringing the immersions to our discussion is that the immersed closed walks, that represent the immersed orbits $C_{j}(\gamma, \boldsymbol{\xi})$ in the decomposition of a $\mathbb{Z}_{2}$-cycle $\gamma$ on a planar graph $\mathcal{G} \subset \mathbb{R}^{2}$ in Lemma IV.8 can be deformed in a natural way to immersions $\bar{C}_{j}: S^{1} \rightarrow \mathbb{R}^{2}$ and studied using topological methods. This rationalizes the terms immersed closed walk and immersed orbit, we have introduced in the context of graphs. We further discuss the self-intersection invariant $p: \operatorname{Imm}\left(S^{1}, \mathbb{R}^{2}\right) \rightarrow \mathbb{Z}_{2}$ that associates with an immersion $C: S^{1} \rightarrow \mathbb{R}^{2}$ the number of self-intersections modulo two. The self-intersection invariant provides a topological interpretation of the intersection number $N(\gamma, \boldsymbol{\xi})$ that appears in Lemma IV.8. In the context of the self-intersection invariant we will refer to Smale-Hirsch-Gromov (SHG) theorem [37] that reduces the problem of studying the topological properties of immersions $C: S^{1} \rightarrow \mathbb{R}^{2}$ to a much simpler problem of studying their phase-space counterparts $f(C): S^{1} \rightarrow \mathbb{R}^{2} \times S^{1}$. In particular, the SHG theorem rationalizes a natural appearance of the phase space $M^{3}=\mathbb{R}^{2} \times S^{1}$. We proceed with introducing the notion of the spinor structure on $\mathbb{R}_{\tilde{C}}^{2}$ viewed as a binary function $s: L M^{3} \rightarrow \mathbb{Z}_{2}$ that associates with any phase-space trajectory $\tilde{C}: S^{1} \rightarrow M^{3}$ a number $s(\tilde{C}) \in \mathbb{Z}_{2}$ and can be also defined for the immersions $C: S^{1} \rightarrow \mathbb{R}^{2}$ by $s(C)=s(f(C))$. The spinor structure provides a topological interpretation of the factors $\varepsilon\left(C_{j}(\gamma, \boldsymbol{\xi})\right)=(-1)^{s}\left(\bar{C}_{j}(\gamma, \boldsymbol{\xi})\right)$ in terms of the value of the spinor structure on the relevant immersions. Finally, we briefly discuss a topological relation between the spinor structure and self-intersection invariant that stands behind the statement of LemmaIV.8.

We start with introducing a concept of an immersion. To that end we consider a particle moving smoothly over a closed trajectory in a plane two-dimensional space $\mathbb{R}^{2}$, where thus smooth planar trajectory $C: S^{1} \rightarrow \mathbb{R}^{2}$ parameterized by $\boldsymbol{r}(t)=\left(r_{1}(t), r_{2}(t)\right.$ where the "time" $t$ belongs to $S^{1}$. The trajectory is called an immersion, which is denoted by $C: S^{1} \leftrightarrow \mathbb{R}^{2}$ if the velocity stays non-zero, i.e., $\dot{\boldsymbol{r}}(t) \neq 0$, at all $t$. In the following the key space will be the iso-energetic shell $M^{3}=\mathbb{R}^{2} \times S^{1}$ of a free planar particle so that for $\boldsymbol{x}=\left(r_{1}(t), r_{2}(t), \theta(t)\right) \in \mathbb{R}^{2} \times S^{1}$, where the angular variable $\theta \in S^{1}$ describes the velocity direction (i.e., the normalized particle velocity) $\dot{\boldsymbol{r}}(t)|\dot{\boldsymbol{r}}(t)|^{-1} \in S^{1}$. Following the terminology, commonly accepted in mathematical physics, we will also refer to the iso-energetic shell as the particle phase space. For a smooth planar trajectory $C$ with a non-zero at all times velocity, i.e., an immersion, we denote by $f(C)$ its phase-space counterpart with the normalized velocity, which clearly forms a trajectory in $M^{3}$ (see Fig. 10 for an illustrative example).

Our next step, as outlined above, is to describe the self-intersection invariant, defined as the modulo two number of self-intersections $i(C)=N(C)(\bmod 2)$ of a smooth planar trajectory $C: S^{1} \rightarrow \mathbb{R}^{2}$. It is intuitively clear that $i(C)$ is a topological invariant only for immersions $S^{1} \rightarrow \mathbb{R}^{2}$, i.e., it stays the same only if we deform a trajectory so that it stays an immersion all the time, since in this case the self-intersections can appear and disappear in pairs only ${ }^{6}$. Immersions can be conveniently studied using the SHG theorem that can be applied for our purposes in the following way. Interpret the procedure of associating with an immersion $C$ its phase space counterpart $f(C)$, as described above, as a map $f: \operatorname{Imm}\left(S^{1}, \mathbb{R}^{2}\right) \rightarrow L M^{3}$ from the space of immersion of a closed trajectory on a plane with everywhere nonzero velocity to the space $L M^{3}$ of closed trajectories in $M^{3}=\mathbb{R}^{2} \times S^{1}$. The SHG theorem, applied to our case, claims that $f$ is a weak homotopy equivalence. In particular it implies that given two immersions, $C, C^{\prime} \in \operatorname{Imm}\left(S^{1}, \mathbb{R}^{2}\right)$, to answer the question whether $C$ can be deformed to $C^{\prime}$, while staying an immersion during the whole deformation, is equivalent to finding out whether $f(C)$ can be deformed to $f\left(C^{\prime}\right)$ without any further restrictions. Note that the SHG theorem explicitly classifies the immersions $S^{1} \rightarrow \mathbb{R}^{2}$ : since the coordinate space $\mathbb{R}^{2}$ is contractible, only the velocity component $\theta_{C}: S^{1} \rightarrow S^{1}$ of the phase-space trajectory $f(C): S^{1} \rightarrow \mathbb{R}^{2} \times S^{1}$ matters, with $\theta_{C}$ being fully homotopically determined by its $\operatorname{degree} \operatorname{deg}\left(\theta_{C}\right) \in \mathbb{Z}$ that describes the total number of rotations the velocity direction makes over the whole trajectory. In particular two immersions $C, C^{\prime} \in \operatorname{Imm}\left(S^{1}, \mathbb{R}^{2}\right)$ are equivalent if and only if $\operatorname{deg}\left(\theta_{C}\right)=\operatorname{deg}\left(\theta_{C^{\prime}}\right)$, i.e. the direction of velocity (the angle) makes the same number of rotations over both trajectories.

Consider concatenation of two trajectories $C_{1} \star C_{2}$ that share the starting point point in $\mathbb{R}^{2}$, understood as trajectory of a particle cycling $C_{2}$ first and then going over the $C_{1}$. The concatenation results in

$$
i\left(C_{1} \star C_{2}\right)=i\left(C_{1}\right)+i\left(C_{2}\right)+C_{1} \cdot C_{2}+1
$$

\footnotetext{
${ }^{6}$ If the condition of having everywhere non-zero velocity is relaxed, this would be not true anymore. This can be illustrated using a simple example. One can imagine a closed trajectory of the shape of "figure eight" from Fig. 10 being deformed in a way that one half of it becomes smaller turning into a point with a cusp, followed by smothering the cusp, which results into a circle. The number of self-intersections changes by one. Note that at the stage of the deformation when we have a cusp, the velocity of the trajectory at a cusp turns to zero. This means that the map $C: S^{1} \rightarrow M^{2}$ at this stage is not an immersion, which actually allows the parity of the self-intersection number to be changed.
} 
where $C_{1} \cdot C_{2}$ denotes the modulo two intersection index (the modulo two number of intersection points) of $C_{1}$ and $C_{2}$. Eq. A1 reflects the fact that the number of self-intersections of a concatenation of $C_{1}$ with $C_{2}$ is the number of self-intersections of $C_{1}$ plus the number of self-intersections of $C_{2}$ plus the number of intersections of $C_{1}$ with $C_{2}$ plus one (the self-intersection at the point of concatenation), which, as opposed to the self-intersection index, is well-defined for any continuous closed trajectories, not necessarily immersions. Note that for the planar case discussed here $C_{1} \cdot C_{2}=0$ (intersections appear and disappear in pairs), stated differently it reflects the fact that all cycles in $\mathbb{R}^{2}$ are contractable. Nevertheless, we keep these contributions, since for our application it will be convenient to treat intersections and self-intersections on equal footing. By defining $p(C)=i(C)+1$ we arrive at

$$
p\left(C_{1} \star C_{2}\right)=p\left(C_{1}\right)+p\left(C_{2}\right)+C_{1} \cdot C_{2} .
$$

By the SHG theorem the topological classes of immersions $S^{1} \rightarrow \mathbb{R}^{2}$ are fully described by the topological classes of their phase space counterparts $f(C): S^{1} \rightarrow M^{3} \cong \mathbb{R}^{2} \times S^{1}$, the latter being labeled by the first homology group $H_{1}\left(M^{3}\right) \cong \mathbb{Z}$, counting the number of rotations of the velocity orientation along the immersion. The self-intersection invariant, therefore, is also defined in terms of the natural factorization $p: \mathbb{Z} \rightarrow \mathbb{Z}_{2}$ (i.e., it can be viewed as a composition $L M^{3} \rightarrow H_{1}\left(M^{3}\right) \rightarrow \mathbb{Z}_{2}$ where the left map associates with a closed phase space trajectory the number of the velocity rotations), and satisfies the property

$$
p\left(\gamma_{1}+\gamma_{2}\right)=p\left(\gamma_{1}\right)+p\left(\gamma_{2}\right)+g\left(\gamma_{1}\right) \cdot g\left(\gamma_{2}\right)=p\left(\gamma_{1}\right)+p\left(\gamma_{2}\right)
$$

where $g(\gamma)$ is a closed trajectory in $\mathbb{R}^{2}$ that is obtained by reduction (projection) from $M^{3}$ by simply ignoring information about orientation of the particle velocity. $g\left(\gamma_{1}\right) \cdot g\left(\gamma_{2}\right)$ stands for the intersection index (mod 2) of the two reduced closed trajectories, and for the planar $\mathbb{R}^{2}$ case considered in the paper it is equal to zero, i.e., $g\left(\gamma_{1}\right) \cdot g\left(\gamma_{2}\right)=0$.

Consider a composite trajectory defined as a union of smooth closed and not necessarily intersecting trajectories (immersions). In this case we define $p\left(\sum_{j=1}^{n} C_{j}\right)=i\left(\bigsqcup_{j=1}^{n} C_{j}\right)+n$, which generalizes the definition of a single smooth trajectory (an immersion). Note that this definition ensures the property given by Eq. A3. Summarizing, we have an invariant $p(\gamma)$ defined on $H_{1}\left(M^{3}\right) \cong \mathbb{Z}$ that satisfies Eq. A3 and if a complex trajectory is decomposed into a set of smooth closed trajectories we derive

$$
p(\gamma)=p\left(\bigsqcup_{j=1}^{n} C_{j}\right)=\sum_{j=1}^{n} i\left(C_{j}\right)+\sum_{j<k} g\left(C_{j}\right) \cdot g\left(C_{k}\right)+n=\sum_{j=1}^{n} p\left(C_{j}\right)+\sum_{j<k} g\left(C_{j}\right) \cdot g\left(C_{k}\right)=\sum_{j=1}^{n} p\left(C_{j}\right)
$$

This completes our discussion of the self-intersection invariant.

We are now in a position to introduce the notion of a spinor structure, as outlined in the beginning if this subsection. A spinor structure can be interpreted as a map $s: L M^{3} \rightarrow \mathbb{Z}_{2}$ that associates with any phase-space trajectory $\tilde{C}: S^{1} \rightarrow \mathbb{R}^{2} \times S^{1}$ its parity $s(\tilde{C})=0,1$. The spinor structure map $s$ should satisfy the following two conditions: (a) Any continuous deformation of $\tilde{C}$ does not change $s(C)$, or more accurately (and generally) if $\tilde{C} \sqcup \tilde{C}^{\prime}$ form a boundary of a two-dimensional domain then $s(\tilde{C})=s\left(\tilde{C}^{\prime}\right)$; and (b) $s\left(\tilde{C}_{\boldsymbol{r}}\right)=1$ for $\tilde{C}_{\boldsymbol{r}}(t)=(\boldsymbol{r}, t)$ being a phase space trajectory with the constant position $r$ and the velocity making a full rotation. Obviously, the spinor structure can be also viewed as a map $s: \operatorname{Imm}\left(S^{1}, \mathbb{R}^{2}\right)$ if we define $s(C)=s(f(C))$ for $C: S^{1} \rightarrow \mathbb{R}^{2}$. For this interpretation the condition (b) means $s\left(C_{\boldsymbol{r}}\right)=1$ where the immersion $C_{\boldsymbol{r}}$ represents a circle on $\mathbb{R}^{2}$, centered at $\boldsymbol{r}$ with a very small radius. Obviously, according to the Stokes theorem applied to the phase space, any spinor structure $s(C)$ can be represented as

$$
s(C)=s(f(C))=\int_{f(C)} A_{j}(\boldsymbol{x}) d x^{j}, \quad \int_{\{\boldsymbol{r}\} \times S^{1}} A_{j}(\boldsymbol{x}) d x^{j}=1,
$$

where $\boldsymbol{A}$ is an Abelian curvature-free vector potential $\boldsymbol{A}$, in the phase space $M^{3}=\mathbb{R}^{2} \times S^{1}$. The curvature-free condition reads $F_{i j}=\partial_{i} A_{j}-\partial_{j} A_{i}=0$, thus expressing the condition (a) formulated above. From the definitions of the spinor structure and the vector potential, one finds that the value $s(C)$ of the spinor structure map on any immersion $C: S^{1} \rightarrow \mathbb{R}^{2}$ is given by the modulo two total number of the particle velocity rotations along the closed trajectory (see Fig. 10 for an illustration). Note that for our planar case the conditions (a) and (b) define a unique function $s$, which means that there is a unique spinor structure on $\mathbb{R}^{2}$ (this is not true in the surface case). Also, this unique spinor structure can be described by different gauge fields $\boldsymbol{A}$ that differ by gauge transformations $A_{j} \mapsto A_{j}+\partial_{j} \phi$. This completes our discussion of the spinor structures. 

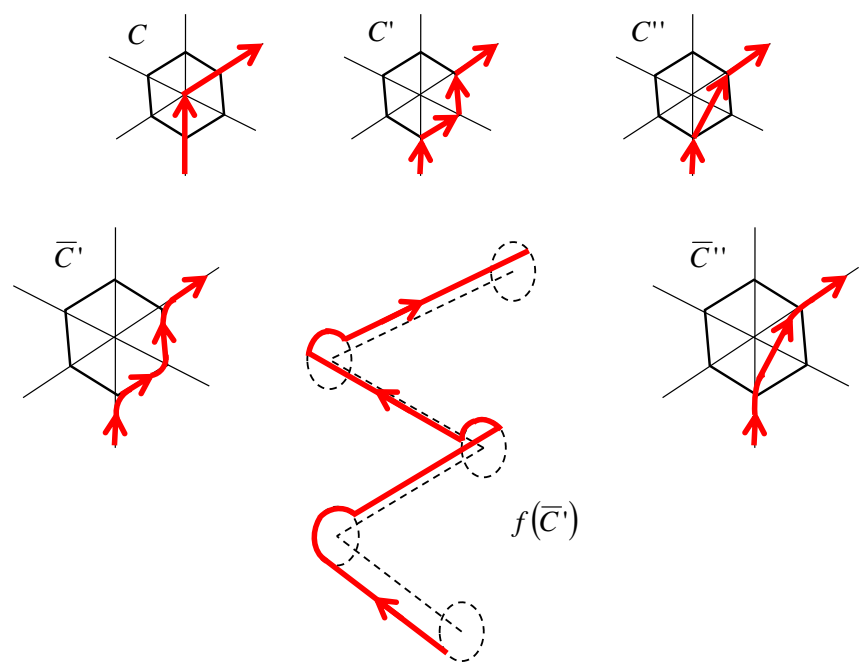

FIG. 11: Schematic illustrations for details of the transformation procedure from an element of a directed orbit on a graph to its particle-trajectory smooth analog embedded into $\mathbb{R}^{2}$ and consequently into $M^{3}$. Notice that if the direction of the orbit is reversed the trajectory $f\left(\bar{C}^{\prime}\right)$ in $M^{3}$ will change. Underlying spinor structure is not shown on the figure.

\section{Topological interpretation of Lemma IV.8}

To demonstrate the equivalence of the combinatorial and topological definitions of $\varepsilon(C)$ we view a directed immersed orbit $C^{\prime}$ on $\mathcal{G}^{e}$, associated with an immersed orbit on $\mathcal{G}$ as a continuous piece-wise smooth trajectory in $\mathbb{R}^{2}$. See Fig. 11 for a schematic illustration of the orbit-to-trajectory transformation. In very small neighborhoods of the nodes we deform $C^{\prime}$ in an obvious way, so that we do not gain self-intersections in these small neighborhoods, to obtain an immersion $\bar{C}^{\prime}: S^{1} \rightarrow \mathbb{R}^{2}$. In the small neighborhoods of the nodes, where the deformations have been performed, the coordinate $\boldsymbol{r}$, i.e., the $\mathbb{R}^{2}$ component of the corresponding phase-space trajectory $f\left(\bar{C}^{\prime}\right): S^{1} \rightarrow M^{3}$ is almost constant, whereas the velocity orientation $\theta$ makes a finite rotation in the counterclockwise or clockwise directions for the left and right turns, respectively. Following $f\left(\bar{C}^{\prime}\right)$ on the deformation of $\bar{C}^{\prime}$ back to $C^{\prime}$ we obtain a continuous piece-wise smooth trajectory in $M^{3}$ that represents a orbit in $M^{3}$, denoted with some minor abuse of notation $f\left(C^{\prime}\right): S^{1} \rightarrow M^{3}$, whose smooth pieces $B_{a b}$ and $B_{a c}^{(b)}$ correspond to the edges and the nodes (velocity turns at the nodes) of $C^{\prime}$, respectively. The value of $s\left(f\left(C^{\prime}\right)\right)$ can be considered as a sum of the contributions from the smooth pieces, labeled by the edges and nodes of $C^{\prime}$, according to Eq. A5. Indeed, we denote by $B_{a b}$ a smooth path in $\mathbb{R}^{2}$ that represents the edge of $\mathcal{G}^{e}$ in the embedding $\mathcal{G} \subset \mathbb{R}^{2}$, whereas for a triplet $a \rightarrow b \rightarrow c$ of $\mathcal{G}^{e}$ we denote by $B_{a c}^{(b)}$ the shortest path on $\left\{\boldsymbol{r}_{b}\right\} \times S^{1}$ that connects the directions of the velocities at $\boldsymbol{r}_{b}$ on the paths $B_{a b}$ and $B_{b c}$, respectively. Then the aforementioned edge and vertex contributions have the form

$$
s_{a b}=s\left(f\left(B_{a b}\right)\right)=\int_{f\left(B_{a b}\right)} A_{j}(\boldsymbol{x}) d x^{j}, \quad s_{a c}^{(b)}=\int_{B_{a c}^{(b)}} A_{j}(\boldsymbol{x}) d x^{j},
$$

respectively. Naturally, $\varepsilon\left(f\left(C^{\prime}\right)\right)=(-1)^{s\left(f\left(C^{\prime}\right)\right)}$ is given by the product of the edge and node contributions and, provided the latter are represented by the Kasteleyn edge orientations and left triplet orientations, which can be always achieved by a gauge transformation of the field $\boldsymbol{A}$, we reproduce the original combinatorial form, i.e., $\varepsilon_{C}(C)=$ $\varepsilon_{T}\left(f\left(C^{\prime}\right)\right)$, where the subscripts $C$ and $T$ stand for combinatorial and topological, respectively.

To evaluate $q(\gamma)$ for a $\mathbb{Z}_{2}$ cycle we decompose it into $n(\gamma, \boldsymbol{\xi})$ immersed orbits $C_{j}=C_{j}(\gamma, \boldsymbol{\xi})$ and consider $C_{j}$ and $C_{j}^{\prime \prime}$ as continuous piece-wise smooth orbits in $\mathbb{R}^{2}$. We denote by $\bar{C}_{j}: S^{1} \rightarrow \mathbb{R}^{2}$ and $\bar{C}_{j}^{\prime \prime}: S^{1} \rightarrow \mathbb{R}^{2}$ the immersions, obtained by slight deformations of $C_{j}$ and $C_{j}^{\prime \prime}$ in small neighborhoods of the nodes of $\mathcal{G}$ and $\mathcal{G}^{e}$, respectively, using the procedure described above in the context of $C^{\prime}$. It is easy to see that $\bar{C}_{j}, \bar{C}_{j}^{\prime}$, and $\bar{C}_{j}^{\prime \prime}$ can be deformed to each other within the immersion space, in particular $f\left(\bar{C}_{j}\right), f\left(\bar{C}_{j}^{\prime}\right)$, and $f\left(\bar{C}_{j}^{\prime \prime}\right)$ represent homologically equivalent cycles in 
$M^{3}$. Therefore, we have

$$
\begin{aligned}
q(\gamma) & =q\left(\bigsqcup_{j=1}^{n} C_{j}\right)=\sum_{j=1}^{n} q\left(\bar{C}_{j}\right)+\sum_{j<k} C_{j}^{\prime \prime} \cdot C_{k}^{\prime \prime}=\sum_{j=1}^{n} s\left(f\left(\bar{C}_{j}\right)\right)+\sum_{j=1}^{n} p\left(f\left(\bar{C}_{j}^{\prime \prime}\right)\right)+\sum_{j<k} C_{j}^{\prime \prime} \cdot C_{k}^{\prime \prime} \\
& =\sum_{j=1}^{n} s\left(f\left(\bar{C}_{j}^{\prime}\right)\right)+n+\left(\sum_{j=1}^{n} i\left(f\left(\bar{C}_{j}^{\prime \prime}\right)\right)+\sum_{j<k} C_{j}^{\prime \prime} \cdot C_{k}^{\prime \prime}\right)=\sum_{j=1}^{n} s\left(f\left(C_{j}^{\prime}\right)\right)+n+N,
\end{aligned}
$$

where $N=N(\gamma, \boldsymbol{\xi})$ is the total number of intersections and self-intersections. Combining Eq. A7 with the relation $\varepsilon_{C}\left(C_{j}\right)=\varepsilon_{T}\left(f\left(C_{j}^{\prime}\right)\right)=(-1)^{s\left(f\left(C_{j}^{\prime}\right)\right)}$ we complete our topological interpretation of the statement of Lemma IV.8.

\section{Topology on Surface Graphs: related approaches and future work}

A topological interpretation of Lemma IV.8 discussed in this Appendix is based on establishing an equivalence between the definitions of $\varepsilon(C)$ in terms of a Kasteleyn orientation on the extended graph Eq. (6) and a spinor structure on $\mathbb{R}^{2}$, respectively. This description allows generalization from $\mathbb{R}^{2}$ to surfaces $M^{2}$ with finite genus. A relation between Kasteleyn orientations on surface graphs $\mathcal{G} \subset M^{2}$ and spinor structures on the surface has been established by Cimasoni and Reshetikhin [34, who demonstrated that a combination of a Kasteleyn orientation and a valid dimer configuration on $\mathcal{G}$ produces a spinor structure on $M^{2}$. We have implemented a very similar, still different approach by associating a spinor structure on $\mathbb{R}^{2}$ with a Kasteleyn orientation on the extended graph $\mathcal{G}^{e} \subset \mathbb{R}^{2}$. In a forthcoming publication we will demonstrate that generalization of our construction solves the problem in the general $M^{2}$ surface case, thus allowing to relate partition function of a general discrete-variables Wick model defined on a graph embedded in a surface of genus $g$ to a sum over $2^{2 g}$ contributions, each associated with a partition function of a fermion model of an allowed equivalence classes of spinor structures of $M^{2}$.

It is also worth pointing out that Cimasoni and Reshetikhin [34 used the aforementioned self-intersection invariant $N(C)$ implicitly since the quadratic form $q$ they have used to study the dimer model on surface graphs and the one considered here (in our planar case $q \equiv 0$ ) are actually the same. However, the objects that naturally arise in the studies of the dimer model on surface graphs are represented by simple unions of non-self-intersecting loops [31, 32, 34, 35], and, therefore, the issue of intersections is never raised in the context of the dimer model. On the contrary, intersections play a key role in establishing the correspondence between the Wick and fermion models, and should be handled explicitly. To the best of our knowledge, in the context of $2 d$ statistical mechanics for the first time the self-intersection invariant, of the type discussed above, however in a gauge of another form, has been brought up by Kac and Ward 10 to come up with an exact solution for the two-dimensional Ising model on a square grid. In the later work, where the Majorana spinors on irregular lattices have been studied [38, 39], the authors have been using the complex valued phase factors $e^{i \theta}$ to establish relations between the free fermion and binary (Ising) models. This can be viewed as an extension of the approach of 10 from a regular to irregular lattices. In this manuscript we have, in a sense, followed [34] by working with the "Kasteleyn" phase factors \pm 1 . The equivalence between the approaches that use different phase factors can be established using a gauge transformation of the field $\boldsymbol{A}$ that describes a spinor structure according to Eq. A5.

\section{Appendix B: Extending the gauge group}

In this Appendix we discuss an alternative way of reducing the three illustrative models, dimer, ice, and Ising discussed in Section $\mathrm{V}$, to the WBG model described by Eqs. (23). In Subsection VA the dimer model has been converted to the WBG model via an example of the gauge transformation described in Eqs. 14]15]16. The ice and Ising models strictly speaking do not belong to the class of vertex models, considered in this manuscript, and an additional geometrical transformation (an extension of the original graph) has been introduced in Sections V CVD .

In this Appendix we demonstrate that the equivalence of the ice model and the Ising model to respective WBG models can be established without extending the original graph. To that end we extend the gauge group (i.e., the group of gauge transformations) by relaxing the constraints given by Eq. 15. Specifically, we relax the constraint $\pi_{a b}=\pi_{b a}$ in the definition of the edge variables, so that the edge variables $\boldsymbol{\pi}_{\alpha}$ are represented by pairs $\boldsymbol{\pi}_{\{a, b\}}=\left(\pi_{a b}, \pi_{b a}\right)$ of binary variables. In addition to the vertex weights $f_{a}\left(\boldsymbol{\pi}_{a}\right)$ we further introduce the edge weights $g_{\alpha}\left(\boldsymbol{\pi}_{\alpha}\right)$. We will also use a notation $g_{a b}\left(\pi_{a b}, \pi_{b a}\right)$ with a natural constraint $g_{a b}\left(\pi_{a b}, \pi_{b a}\right)=g_{b a}\left(\pi_{b a}, \pi_{a b}\right)$ that makes this notation consistent 
with the original one. The partition function of such an Extended EBG, thus EEBG, becomes

$$
Z_{E E B G}=\sum_{\boldsymbol{\pi}} \prod_{a \in \mathcal{G}_{0}} f_{a}\left(\boldsymbol{\pi}_{a}\right) \prod_{\alpha \in \mathcal{G}_{1}} g_{\alpha}\left(\boldsymbol{\pi}_{\alpha}\right)
$$

A gauge transformation of an extended model is described by a set $\left\{G_{a b}\left(\pi_{a b}, \pi_{a b}^{\prime}\right)\right\}_{\{a, b\} \in \mathcal{G}_{1}}$ of local invertible $2 \times 2$ matrices with no other restrictions. A gauge transformation for the vertex functions is still given by Eq. (14), whereas the edge functions are transformed according to the rule

$$
g_{\{a, b\}}\left(\boldsymbol{\pi}_{\{a, b\}}\right) \rightarrow \tilde{g}_{\{a, b\}}\left(\boldsymbol{\pi}_{\{a, b\}}\right)=\sum_{\pi_{\{a, b\}}^{\prime}} G^{a b}\left(\pi_{a b}, \pi_{a b}^{\prime}\right) G^{b a}\left(\pi_{b a}, \pi_{b a}^{\prime}\right) g_{a b}\left(\pi_{a b}^{\prime}, \pi_{b a}^{\prime}\right)
$$

where $G^{a b}$ denote the matrices inverse to $G_{a b}$, i.e.,

$$
\sum_{\pi_{a b}} G^{a b}\left(\pi_{a b}^{\prime \prime}, \pi_{a b}\right) G_{a b}\left(\pi_{a b}, \pi_{a b}^{\prime}\right)=\delta\left(\pi_{a b}^{\prime \prime}, \pi_{a b}^{\prime}\right)
$$

Obviously the partition function of the extended model is invariant with respect to the extended gauge transformations.

We further note that the EBG model $\sqrt{16}$ ) is a particular case of the EEBG model (B1) that corresponds to the choice of the edge functions in a form

$$
g_{a b}\left(\pi_{a b}, \pi_{b a}\right)=\delta\left(\pi_{a b}, \pi_{b a}\right)
$$

and the EBG-model gauge transformations, i.e., the ones that satisfy the constraints of Eq. (15), can be viewed as the extended gauge transformation that preserve the edge functions of the form, given by Eq. (B). Obviously, any extended model is equivalent to an EBG model.

The ice model represents a class of graph-based models, hereafter referred to as arrow models on graphs (AG), where a configuration is given by a graph orientation, i.e, a set of arrows associated with the non-oriented edges, rather than binary variables residing on the edges. Any AG model can be viewed as a particular case of EEBG model by associating with a local edge configuration of an AG model on $\{a, b\}$, determined by an arrow $a \rightarrow b$, the edge configuration $\pi_{a b}$ with $\pi_{a b}=0$ and $\pi_{b a}=1$. Obviously the obtained EEBG model corresponds to the choice of the edge functions represented by the same off-diagonal matrix with $g_{a b}(0,0)=g_{a b}(1,1)=0$ and $g_{a b}(0,1)=g_{a b}(1,0)=1$. A homogeneous gauge transformation of this form is

$$
G^{a b}=\frac{1}{\sqrt{2}}\left(\begin{array}{cc}
1 & 1 \\
i & -i
\end{array}\right)
$$

transforms the edge functions $g_{\alpha}$ of an AG model into respective ones described by Eq. (B4). This establishes an equivalence between an AG model and respective EBG model. Note that the edge function for an AG model is symmetric and, therefore, can be viewed as a quadratic form. Then the gauge transformation can be interpreted as the quadratic form diagonalization. If performed over real numbers the result has a diagonal form with the signature $(1,-1)$. The imaginary units in the matrix elements in Eq. (B5) are responsible for transforming the obtained diagonal matrix into the form given by Eq. (B4).

Consider a subclass of AG models, hereafter referred to as even AG models, whose vertex functions stay invariant upon a change the direction of all arrows, associated with the vertex. (Note that the ice model belongs to this subclass.) It is easy to show that the described above homogeneous gauge transformation transforms an even AG model into an even EBG model. Also note that in this even case the vertex functions of the resulting even EBG model are real, despite of the presence of the imaginary unit in the gauge transformation given by Eq. (B5). The suggested gauge transformation identifies the subclass of AG models (including, e.g., the ice model) resulting in WBG models upon the gauge transformation given by Eq. (arrow-to-EBG), and, therefore are easy.

The Ising model on an arbitrary graph $\mathcal{G}$ can be also viewed as an EEBG model with the vertex and edge function given by

$$
\begin{aligned}
& f_{a}(\boldsymbol{\pi})=\left\{\begin{array}{l}
1, \\
0 b, c \in \delta_{\mathcal{G}}(a) \quad \pi_{a b}=\pi_{a c},
\end{array},\right. \\
& g_{a b}\left(\pi_{a b}, \pi_{b a}\right)= \begin{cases}\gamma, & \pi_{a b}=\pi_{b a} \\
\mu, & \pi_{a b} \neq \pi_{b a}\end{cases}
\end{aligned}
$$

which differ from Eqs. (30) and (31) actually by notations only. The gauge transformation that diagonalizes the vertex functions $g_{\alpha}$, viewed as quadratic forms, turns the Ising model into an even EBG model that belongs to the WBG subclass, as outlined in subsection VD 
Finally, we note that the approach discussed in this Appendix, based on the extended gauge transformation, can be viewed as an alternative formulation leading to the well-known high-temperature expansion of the Ising model, when the expansion is interpreted as a loop series with only $\mathbb{Z}_{2}$-cycles providing non-zero contributions. 